shop — qui permet au syndicat majoritaire d'avoir l'exclusivité de la représentation, et donc de choisir ses membres; elle doit affronter le militantisme des patrons qui sont partisans acharnés de la liberté du contrat et hostiles à la reconnaissance syndicale.

Aussi, en 1920, le bilan syndical est-il contrasté. Collomb peut affirmer sans crainte que l'AFL n'a non seulement pas privilégié l'intégration des immigrés, mais choisi de participer à la définition de la nation américaine plutôt que de défendre la classe ouvrière. Elle est parvenue à ses fins en faisant de la fermeture des frontières un enjeu national et en s'attaquant à tout mouvement concurrent. Cela explique les limites de l'essor du parti socialiste, ou l'échec des IWW, mais, ce faisant, la grande centrale américaine édifie sa force sur l'exclusion et le conservatisme. Comme toujours aux États-Unis où le pire n'est pas toujours sûr, il existe des syndicats de l'AFL qui sauvent les principes et parviennent à se montrer plus dignes de leur mission d'intégration de tous les travailleurs par la classe et la nation, mais ils forment l'exception qui confirme la règle.

Cette imparable démonstration repose sur des sources inattaquables et sur une analyse rigoureuse et nuancée, qui ne fait pas disparaittre certaines redites, en raison d'un plan complexe. Il faut lire ce livre, si l'on s'intéresse au mouvement social : il montre toute la redoutable ambiguïté d'un certain modèle américain.

Jacques PoRTes

\title{
HISTOIRE DES SCIENCES
}

Aline Rousselle, La Contamination spirituelle. Science, droit et religion dans l'Antiquité. Paris, Les Belles Lettres, 1998. $15 \times$ 21,5, 375 p., index (Histoire).

La Contamination spirituelle d'Aline Rousselle se propose de revenir sur les ruptures et les continuités qui ont marqué la conversion de l'Antiquité. L'auteur étudie successivement la science, le droit et la religion afin de démontrer que le christianisme s'est largement nourri des connaissances scientifiques et des dispositions juridiques de son temps. Rassemblant des articles publiés depuis 1980, les trois parties parviennent à construire une démonstration cohérente que ne remettent pas en cause, chemin faisant, quelques répétitions.

La première partie retrace l'évolution des connaissances médicales, notamment anatomiques, à travers le corpus hippocratique et les œuvres des grands médecins de l'Antiquité (Soranos, Galien, Oribase, etc.). Ces documents ne sont pas des disserta- 
tions abstraites : les traités cnidiens, par exemple, qui étudient la sexualité féminine, accordent une place importante aux témoignages individuels. Recueillant les récits de ces femmes qui parlent de leur corps, ces textes scientifiques transmettent ainsi « une tradition orale féminine ». Certaines de leurs conclusions ont été vivement critiquées : le chapitre $\mathrm{I}$, «Corps féminin et hystérie», rappelle par exemple que l'hypothèse d'un "sperme féminin », soutenue par la tradition hippocratique, fut vigoureusement combattue par Aristote qui refusait de subordonner la procréation à l'émission d'une semence féminine (chapitre II). L'examen méthodique de ces débats révèle toutefois la validité de certaines observations. La dissection et la vivisection ont notamment permis à Galien, au II siècle après Jésus-Christ, de comprendre les composants anatomiques de la voix et de les associer aux mécanismes respiratoires (chapitre III). Ces résultats sont d'autant plus intéressants qu'ils traduisent les préoccupations sociales, juridiques et religieuses de leur temps : ainsi, les recherches sur la sexualité féminine reflètent-elles la hantise de la stérilité, à Rome, où la reproduction du corps des citoyens est un enjeu majeur de la politique impériale. De même, les observations sur la voix illustrent l'importance sociale de la rhétorique qui suppose l'éducation de la parole mais aussi la maîtrise des mécanismes de la voix. S'appuyant sur la confrontation des sources littéraires, historiques et scientifiques, l'argumentation précise de A. Rousselle parvient à montrer que «les problèmes sociaux étaient de bons leviers pour la science ». Néanmoins, le style résolument technique de certains développements savants pourra déconcerter les lecteurs auxquels l'utilisation d'un dictionnaire anatomique sera parfois utile.

La deuxième partie aborde ensuite l'évolution juridique de l'Empire. L'auteur cherche à établir que le droit romain, qui allait réglementer, à partir de 212 , le comportement de peuples d'origine ethnique différente, se déterminait moins en fonction de principes abstraits que de «situations réelles », notamment en matière familiale. Le chapitre Iv, inédit, tente d'examiner « la nature du citoyen » qui soustend cette législation, en étudiant le mode de transmission de la citoyenneté romaine. Le renouvellement de la cité a rendu nécessaire l'assimilation de personnes étrangères par un mariage légitime. Il reposait donc sur l'idée d'une nature juridique et non pas biologique. Le chapitre v suivant, "Concubinat et adultère », retrace la genèse des interdits qui encadrent la sexualité, et l'élaboration de la notion de souillure, strictement définie par le droit de l'Empire. Si le citoyen pouvait jouir en toute liberté des faveurs des esclaves, des prostituées, des comédiennes ou des femmes qui avaient perdu leur honorabilité, il ne pouvait convoiter l'épouse d'un autre citoyen sans risquer l'infamie que donne l'adultère. À partir de définitions précises (par exemple, l'homme adultère n'est pas l'infidèle mais l'amant d'une femme mariée), A. Rousselle délimite le champ de la liberté sexuelle en montrant qu'il est au cœur du fonctionnement de la société romaine. En effet, la souillure sexuelle ne se réduit pas à une condamnation morale. Elle traduit l'exclusion de la communauté des citoyens en privant l'infâme de son droit de parole devant les assemblées. Un corps impur n'a plus sa place dans le Corps civique. Les interdits qui pèsent sur les comportements sexuels sont donc bien antérieurs au christianisme. Ils sont définis par une législation qui, indépendamment de toute considération morale, cherchait d'abord à garder le contrôle des naissances citoyennes (chapitre vI : « Statut personnel et usage sexuel dans l'Empire romain »). Cette analyse rappelle au lecteur une caractéristique fondamentale de l'Antiquité romaine : «Tout est conditionné par le 
statut, par des fixations juridiques bien apparentes dans le droit. » Néanmoins, la rigueur de l'arsenal législatif de l'Empire n'excluait pas une certaine souplesse qui favorisa l'assimilation de communautés vivant sous d'autres droits. «La pratique familiale polyjuridique des citoyens romains juifs » (chapitre vII) révèle par exemple que cette souplesse fut facilitée par l'établissement préalable de règles identiques : le témoignage de Flavius Josèphe nous apprend que malgré certaines divergences (la définition de l'inceste, le statut de la polygamie), ces deux législations avaient le même souci du renouvellement de la communauté. Ainsi la Michna juive et le Digeste romain condamnent-ils de la même façon l'homosexualité dont la stérilité menace la reproduction de la communauté. Ce chapitre permet également de constater que la christianisation de l'Empire s'accompagna de règles parfois incompatibles avec les droits locaux (interdiction radicale de la polygamie, limitation de la répudiation de l'épouse), remettant en cause la souplesse de ces pratiques polyjuridiques.

Néanmoins, l'introduction de la troisième partie, consacrée à la religion, précise d'emblée que le christianisme a beaucoup hérité des siècles précédents : la compréhension des canons de la foi, l'organisation de l'Église et la progressive définition d'une spiritualité chrétienne sont étroitement liées aux « élaborations scientifiques et juridiques étudiées dans les chapitres antérieurs ». Le chapitre viII décrit les fondements d'une «cuisine spirituelle » très influencée par les connaissances médicales de l'Antiquité. Alors que les sciences païennes ont établi un lien entre la bonne chère et l'appétit sexuel, les témoignages sur les monachismes martinien et provençal prétendent également que l'apprentissage de la continence suppose une alimentation frugale. Donnant une idée précise de l'alimentation des moines, ils prouvent que les premières règles de la fin $\mathrm{du} \mathrm{IV}^{\mathrm{e}}$ siècle se sont largement inspirées des traités scientifiques qui ont tenté, à leur manière, de maîtriser les caprices du corps. Elles reprennent, par exemple, l'opposition des traitements médicaux (régime $\mathrm{sec}$ et froid/régime humide et chaud) que l'on retrouve chez Oribase, médecin du $\mathrm{IV}^{\mathrm{e}}$ siècle. La littérature ascétique révèle également les difficultés que rencontraient les moines, en Gaule, pour suivre les régimes alimentaires de leurs modèles orientaux, trop austères. Bien que persuadé qu'il est « impossible d'éteindre les feux de la concupiscence sans réfréner les plaisirs du ventre», Césaire d'Arles doit reconnaître, dans sa Règle aux moines, que "si l'excès de bonne chère est chez les Grecs gloutonnerie, chez les Gaulois, c'est appétit naturel ». Il faut noter toutefois que la lecture de ce chapitre est rendue difficile par une erreur informatique qui a décalé la numérotation des notes à partir de la page 208. Le chapitre IX suivant propose une «étude positive des miracles chrétiens de la fin $\mathrm{du}_{\mathrm{IV}}^{\mathrm{e}}$ siècle ». Le mot miracle a permis d'unifier les faits étonnants en leur conférant une origine divine. Cherchant à dépasser cet obstacle épistémologique, A. Rousselle envisage « un nouveau découpage conceptuel» qui vise à montrer que «le miracle est du réel: ce n'est pas de la représentation ». Ainsi, le miracle de saint Martin, immobilisant une procession païenne, pourrait-il s'expliquer par un geste d'hypnose. S'appuyant sur les connaissances de la technique hypnotique dans l'Antiquité, cette hypothèse hardie ne saurait remplacer les analyses spirituelles (imitation du Christ), pastorales (christianisation du monde païen) et typologiques du miracle (topique de la littérature hagiographique). Toutefois, les intentions de A. Rousselle sont très claires : il ne s'agit pas de comprendre les miracles mais d'expliquer le phénomène qu'ils recèlent. L'auteur examine ensuite la place des femmes dans une société qui ne leur 
reconnaissait pas le pouvoir thaumaturgique (chapitre x). S'il est vrai que, dans la Gaule tardive, «les femmes ne font pas de miracle», certaines fonctions leur confèrent un rôle social important : les prêtrises féminines, le culte et la diffusion des reliques ou encore les soins donnés aux femmes qui venaient, en pèlerinage, sur les tombes des martyrs. L'exemple de ces centres de guérisons (« l'alternative chrétienne aux sanctuaires des sources ») montre, une nouvelle fois, que la conversion de la culture antique n'a pas été vécue comme une rupture dans la mesure où elle a conservé de nombreuses habitudes du monde païen. Cette constatation est encore illustrée par le chapitre XI et dernier, «Le sexe et la parole»: l'élaboration des normes chrétiennes a préservé le lien entre l'intégrité corporelle et la capacité à dire la vérité, c'est-à-dire entre le comportement sexuel et le droit à la parole. Et pour rappeler ce que le christianisme doit à son temps, l'ouvrage se termine sur un parallèle saisissant entre les dispositions conciliaires et la législation impériale. Cette juxtaposition révèle une continuité juridique en ce qui concerne les règles d'incapacités civiques, la définition des différents types d'infamie ou encore l'esclavage: «Comme les Empereurs, les évêques réunis en conciles (concile d'Elvire en 306 et concile d'Epaone en 507) estimèrent qu'il était plus grave pour une femme et pour un homme de commettre l'adultère que de tuer un esclave. »

Ainsi La Contamination spirituelle parvient-elle à montrer que le christianisme s'est nourri des connaissances scientifiques et des dispositions juridiques de son époque. L'élaboration de la notion de souillure est exemplaire : elle doit beaucoup à l'évolution des sciences, même si « le besoin de fixer des règles sexuelles apparaît comme une constante de l'humanité ». La nouvelle religion avec laquelle s'est peu à peu confondu l'Empire n'a donc pas opéré de rupture brutale; elle ne s'est pas non plus réduit à l'héritage d'un passé. C'est pourquoi l'auteur peut conclure sans ambiguiité que la christianisation de l'Antiquité fut profondément «marquée à la fois par les obsessions pluriséculaires d'une société méditerranéenne, par les réponses que la science leur avait apportées, et par les règles que le droit en avait tirées ». Le christianisme fut d'abord un produit de son temps.

Stéphane GioAnNI

Joëlle Ducos, La Météorologie en français au Moyen Âge (XIII ${ }^{e}-{ }{ }^{e}{ }^{e}$ siècles). Paris, Champion, 1998. 15,5 × 22,5, 494 p., bibliogr., index (Sciences, techniques et civilisations du Moyen Âge à l'aube des Lumières, 2).

Le ciel, ses humeurs et ses changements ont toujours captivé l'attention des hommes. Ils y ont cherché des signes, des symboles et des présages. Le Moyen Âge ne déroge pas à cette règle et s'inscrit dans une longue histoire dont l'Antiquité classique a fourni les fondements anciens.

Prévenons d'emblée le lecteur : il s'agit ici d'histoire savante. On ne trouvera pas de jolis parcours pittoresques (et légèrement condescendants envers le sujet traité) dans les savoirs populaires, dans les proverbes et les dictons qui fleurent bon les campagnes traditionnelles, réservoirs de sagesse. Le «rude pueple» comme 
l'évoque un des auteurs étudiés par Joëlle Ducos n'est guère sollicité et sur les 494 pages de l'ouvrage, seules une dizaine d'entre elles en parlent, surtout pour constater qu'il s'agit le plus souvent de citations livresques, ou de brèves allusions au savoir technique, comme celui des marins par exemple.

Qui dit science au Moyen Âge dit réflexion fondée sur les textes légués par les Grecs et les Romains (les « autorités antiques »), et confrontée au savoir religieux (l'Écriture sainte et les œuvres des pères de l'Église qui forment les autorités par excellence). Cette réflexion s'ordonne impérativement en plaçant au-dessus de toutes les sciences, celle de Dieu et la Révélation pour leur donner finalité et sens profond.

J. Ducos retrouve ce débat permanent à propos de la météorologie comme objet scientifique, étude du ciel et des phénomènes qui s'y déroulent. Une sorte de paradoxe car dans les problématiques scientifiques de l'époque, le savant travaille à retrouver et à ordonner les lois de l'Univers et celles de Dieu, le Ciel par excellence, mais, dans ce cas présent, il prend pour objet des phénomènes changeants, imprévisibles et instables, qu'encadrent mal les lois immuables et universelles.

La période envisagée, XIII $^{\mathrm{e}}$ et $\mathrm{XIV}^{\mathrm{e}}$ siècles, représente un moment fort dans l'histoire de la pensée occidentale et plus particulièrement, dans celle qui s'épanouit à Paris, au sein des milieux intellectuels formés à l'Université. Une période qui a vu l'apport de nouveaux textes antiques (grâce aux nombreuses traductions entreprises au siècle précédent en Espagne ou en Italie, suscitées par l'appétit de découvrir des textes antiques connus auparavant seulement par leurs titres ou quelques allusions), qui s'épanouit avec la rédaction des grandes sommes et des encyclopédies, témoins tangibles d'une féconde activité intellectuelle. Une période de confiance et d'optimisme favorable à la réflexion constructive, à l'élaboration de grands systèmes et de grandes synthèses, qui se prolonge jusqu'au milieu du XIv ${ }^{\mathrm{e}}$ siècle. Le sujet du livre de J. Ducos s'inscrit dans ce vaste contexte et se précise en mettant l'accent sur les œuvres en français, c'est-à-dire sur des textes savants traduits du latin en langue dite vulgaire ou sur des textes écrits directement en français, donc tous accessibles aux laïcs qui n'ont pas été formés dans les écoles et qui ne dominent pas la langue latine. Ces textes savants en français marquent une réelle politique d'élargissement culturel reposant en grande part sur une volonté royale que le règne de Charles $\mathrm{V}$ a portée à son maximum.

Le texte de base, dans cette étude, est un livre d'Aristote, Les Météorologiques, connu à partir de deux traductions : une ancienne (datant de la fin du XII siècle), œuvre de Gérard de Crémone qui a travaillé à partir d'une version en arabe venue du texte grec initial; une seconde traduction, dite nouvelle, a été faite par Guillaume de Moerbeke vers 1260 à partir du grec. Le texte est ensuite (à la fin du XIII siècle) traduit en français par un clerc normand, Mathieu le Vilain. À cette base, J. Ducos adjoint les encyclopédies du XIII ${ }^{\mathrm{e}}$ siècle (la partie de ces ouvrages qui concerne le sujet) et en retient quatre, le Sidrach, L'Image du monde, livre de Gossouin de Metz, Le Livre du trésor de Brunet Latin et Le Dialogue de Placides et Timeo. Le XIII ${ }^{\mathrm{e}}$ siècle a produit beaucoup de livres rassemblant et classant toutes les connaissances pour les répandre, mais tous n'accordent pas à la météorologie comme partie de la physique et de l'astronomie une place importante. Les textes du XIV siècle, souvent non édités, ne sont pas tous sollicités pour nourrir le corpus. J. Ducos a choisi d'appuyer ses recherches sur deux livres. Une traduction en français du De proprie- 
tatibus rerum de Barthélemy l'Anglais qui a écrit, en latin, son encyclopédie entre 1230 et 1240, et une traduction de Problèmes due à Evrart de Conty. Ce dernier livre traduit est attribué à Aristote; il s'agit d'un long recueil de questions regroupées par thèmes. Évidemment, J. Ducos dépasse largement ce corpus de base pour mener ses analyses et enrichir ses commentaires. Il n'est pas dans ses intentions de critiquer les théories d'Aristote et l'adaptation qu'en firent les penseurs et vulgarisateurs médiévaux. Le lecteur trouvera des chapitres (II, IV) qui résument et expliquent l'origine des vents, de la pluie, des orages, en suivant les œuvres savantes et les encyclopédies.

Quelques apports du beau livre de J. Ducos peuvent être soulignés.

Le premier, qui revient plusieurs fois au long de la démonstration, concerne les questions de traductions et de vocabulaires. On suit volontiers l'auteur lorsqu'elle explique l'importance du choix de type de traduction : le mot à mot, au risque de décourager le lecteur mais qui ne «trahit» pas la pensée, ou l'adaptation, plus lisible pour ceux qui ne peuvent avoir recours au texte dans sa langue d'origine mais qui peut «déformer » radicalement le texte initial. Aristote était le plus souvent traduit littéralement car il avait le statut «d'autorité » et, comme les textes religieux, devait être cité le plus exactement possible. Dans tous les cas, il a fallu enrichir le vocabulaire français et ce travail d'ajout de mots abstraits, de termes généraux, a permis une rationalité, base d'une réflexion scientifique. Les rédacteurs d'encyclopédies en français ont dû, eux aussi, imaginer tout un vocabulaire et ont contribué de la sorte à cette propédeutique scientifique que constitue un vocabulaire dominé et adapté à son objet.

Ces analyses de mots (rassemblés en annexes finales) prouvent aussi que les penseurs médiévaux ne se contentaient pas de recopier servilement les autorités qu'ils citaient. C'est là un autre apport important du livre. Il montre que les auteurs mêlaient leurs propres réflexions, adaptaient et transformaient ce qui, dans les théories anciennes, ne leur semblait plus utile ou exact. L'admiration proclamée n'a pas tari l'intelligence. La démonstration que fait J. Ducos à propos de la météorologie s'inscrit dans une recherche globale qui ne néglige plus l'histoire de la pensée médiévale, qui ne se borne plus à de rapides caricatures, qui en réhabilite l'intérêt et les enrichissements qu'elle a légués.

Un autre apport du livre tient précisément à ce qu'a de scientifique cette constitution de la météorologie en français comme objet d'étude. Il est défini, nommé, décrit. Il s'agit de comprendre, d'inscrire ces connaissances dans celles de la physique, des lois du monde d'ici-bas, dans celles du ciel. Nul aspect pratique n'est revendiqué ou recherché. Comme toute science, la météorologie est une science désintéressée, attirée vers le haut, le spirituel ou, à tout le moins, la théorie. Elle ne peut se confondre avec le savoir des hommes de mer ou des jardiniers, mais en même temps, elle risque de se couper de l'apport de l'empirique ou des savoirs oraux.

Au tournant du XIII et du XIV ${ }^{\mathrm{e}}$ siècle, les textes encyclopédiques révèlent au mieux à la fois les avancées de la recherche intellectuelle et les butoirs qui l'arrêtent. En mêlant dans leurs livres les citations d'autorités diverses, ces auteurs polygraphes n'évitent pas les opinions contradictoires et les incohérences. Mais ces heurts expriment aussi une rencontre féconde entre pensées différentes, à commencer par celle apportée par les livres d'Aristote. Les réponses à ce dilemme - suivre Aristote 
et sa science ou la Bible et la foi — ont libéré de grands courants de la recherche et de la rationalité, comme en témoignent les œuvres de Thomas d'Aquin ou d'Albert le Grand, comme les diffusent l'encyclopédie de Vincent de Beauvais ou celle de Thomas de Cantimpré. Mais en plaçant systématiquement la théorie et le spirituel comme seuls dignes d'efforts véritables, la réponse intellectuelle du xiII ${ }^{\mathrm{e}}$ siècle a méprisé l'expérience, le monde matériel. Les connaissances nautiques et astronomiques des navigateurs et des marins n'occupent pas leur juste place. Elles sont présentes comme par effraction dans un monde intellectuel qui s'enorgueillit de rester abstrait et général. Sans aucun doute, c'est là une des limites majeures de l'effort intellectuel et des ambitions du monde des savants du Moyen Âge.

Simone Roux

Histoire des sciences arabes. Vol. I : Astronomie, théorique et appliquée; vol. II : Mathématique et physique; vol. III : Technologie, alchimie, sciences de la vie. Éd. sous la dir. de Roshdi Rashed, avec la collab. de Régis Morelon. Paris, Seuil, 1997. $17 \times 22,376$ p., 422 p. et 321 p., pl., ill. (Science ouverte).

Ces trois volumes marquent sans aucun doute un moment important dans l'histoire de l'histoire des sciences. Il s'agit d'abord d'un ouvrage de référence, qui offre, outre les résultats factuels des recherches d'historiens de premier ordre et des analyses originales, une perspective de lecture des sciences arabes qui deviendra elle-même objet historique. L'ensemble n'est pas monolithique, au contraire : à la diversité des sujets répond la diversité des auteurs. Diversité de sensibilité intellectuelle - il ne serait pas très difficile de reconstituer le pedigree théorique de la plupart des contributeurs -, mais aussi différence de valeur, car si l'ensemble est excellent, certaines contributions sont plus excellentes que d'autres. Cependant, sous la houlette épistémologique de Roshdi Rashed, l'ouvrage se présente bel et bien comme un organisme unique, en affichant des normes et un but théoriques communs. Je voudrais faire trois remarques, d'objets et de portées fort différents, à propos de ces trois volumes.

La première remarque porte sur la première note de la première page du premier volume. La science arabe, précise Rashed, est la science écrite en arabe, comme la science grecque est la science écrite en grec. À strictement parler, les choses sont moins simples. La science byzantine est écrite en grec, mais elle ne fait pas partie de la «science grecque». De même les traités scientifiques du XVII ${ }^{\mathrm{e}}$ siècle, écrits en latin ne font pas partie de la «science latine». Qu'on le veuille ou non, le concept de « science arabe » (ou « grecque », ou « latine »...) présuppose un découpage à la manière hégélienne de l'histoire de l'esprit: la science grecque est celle qui a été produite dans - et, peut-être aussi, aux confins de - ce que nous appelons le monde grec antique. On considère généralement que ce monde grec survit au désastre politique que fut la domination romaine, mais qu'il cesse avec le début du «Moyen Âge ». La science arabe est donc plus précisément la science produite, 
principalement, mais pas seulement, en arabe - car qui contesterait que certains textes rédigés en persan ou en syriaque appartiennent à la science arabe? - , par des savants exerçant leur activité à l'intérieur de — ou en relation avec — une entité culturelle que nous appelons la « civilisation arabe». On voit donc fort bien quand commence la science arabe : vers le viII siècle de l'ère chrétienne, l'aire culturelle arabe est une entité historique constituée qui produit des activités scientifiques et techniques originales. Mais quand finit-elle? Ce ne sont pas les critères qui nous ont permis de marquer le commencement de la science arabe que nous pourrons employer pour en marquer le terme. L'histoire de la science arabe finit non pas avec la fin $\mathrm{du}$ «monde arabe » comme la science grecque (faudrait-il dire « antique »?) finit avec le «monde antique », mais quand elle est «surclassée » par la science européenne à partir du XVII ${ }^{\mathrm{e}}$ siècle. Bref, on aurait le plus grand mal à fonder rigoureusement la notion même de «science arabe », et il faut sans doute savoir gré aux auteurs de ne l'avoir point tenté, pour s'en tenir à l'évidence empiriquement constatable de cette production scientifique immense, originale, historiquement déterminante dans l'histoire des progrès de l'esprit humain.

Ma deuxième remarque concerne l'une des appréciations générales que l'on peut porter sur la science arabe, et elle pourrait être induite du plan même de l'ouvrage. Plan en trompe-l'œil, d'une certaine manière. Le premier volume traite de l' " astronomie, théorique et appliquée », à quoi il faut ajouter la considération, indispensable, des effets des sciences arabes dans les régions périphériques et les autres aires culturelles. Sur ce dernier point, l'article de Juan Vernet et Julio Samso sur les développements de la science arabe en Andalousie - au sens de al-Andalus - et celui de Bernard Goldstein sur l'utilisation de la science arabe par les savants juifs médiévaux ne s'en tiennent pas à la seule astronomie, pour des raisons évidentes tenant à la taille de l'ensemble, alors que l'article de Henri Hugonnard-Roche se réduit, si l'on peut dire, à l' «Influence de l'astronomie arabe en Occident médiéval ». Quant à l'astronomie appliquée, il s'agit d'une part de la construction d'instruments comme les globes célestes et les astrolabes étudiée par Francis Maddison et de l'utilisation de l'astronomie pour des fins socialement importantes comme la détermination de la direction de La Mecque pour la prière, la gnomonique et la mesure du temps - trois pratiques étudiées par David King sous le titre un peu ambitieux de «Astronomie et société musulmane » - , et d'autre part de techniques utilisant l'astronomie, comme la cartographie considérée par Edward Kennedy, et la science nautique, qui fait l'objet d'une étude passionnante due à Henri Grosset-Grange et Henri Rouquette. Le deuxième volume s'intitule "Mathématique et physique ». Après une revue des disciplines mathématiques «dures », allant de l'arithmétique à la trigonométrie (six chapitres), et un chapitre sur «L'influence des mathématiques arabes dans l'Occident médiéval » dû à André Allard, on trouve un chapitre sur la science musicale, un autre sur la statique et deux chapitres sur l'optique. Le troisième volume rassemble, enfin, tout le reste : technologie, géographie, botanique et agriculture, alchimie, médecine. Les articles y sont tous bons, parfois excellents, dans des styles très différents : de l'essai brillant d'André Miquel sur la géographie - sans une seule note, mais il n'est pas le seul dans ce cas — à l'exposé truffé de longues citations d'Emilie Savage-Smith sur la médecine - exposé plus qu'honorable, étant donné la difficulté à maîtriser un sujet aussi vaste et aussi contrasté. Le tout finit par un article, enthousiaste et utile, de Françoise Micheau sur « Les institu- 
tions scientifiques dans le Proche-Orient médiéval », et un article sur les « Classifications des sciences » par Jean Jolivet, qui examine les classifications d'al-Kindi, alFarabi et Avicenne. C'est une bonne idée d'avoir mis cette contribution riche et subtile à la fin de cette somme d'histoire des sciences, donnant ainsi à la philosophie le mot de la fin mais pas celui du début.

Pourquoi ai-je parlé, sans intention péjorative aucune, de «trompe-l'œil » ? Parce que mettre l'astronomie en tête peut porter à une erreur de perspective. Certes, la raison incontournable que l'on peut invoquer pour justifier l'ordre adopté, c'est qu'il s'est constitué — ou plutôt qu'il a commencé de se constituer - une astronomie arabe avant que ne commence à se constituer une mathématique arabe. Mais, au vu de la disposition même des articles, l'historien des sciences grecques ne peut guère s'empêcher, surtout s'il a en tête les classifications des sciences étudiées par Jean Jolivet, toutes fortement teintées d'aristotélisme, de penser que les deux premiers volumes de cet ouvrage traitent finalement de ce qu'Aristote appelle les mathématiques et «les plus physiques des sciences mathématiques». Avec, certes, une exception — d'ailleurs partielle — concernant l'optique physiologique, à propos de laquelle Gül Russell rappelle le rôle éminent d'Ibn al-Haytham (l'Alhazen des Latins). Autrement dit, quand le deuxième volume s'intitule « Mathématique et physique », il faudrait s'entendre. Cette physique-là n'est-elle pas constituée d'un ensemble de disciplines directement issues des mathématiques, de sorte que, dans sa structure fondamentale et d'un point de vue bachelardien qui est inévitablement le nôtre - y compris pour les plus continuistes et les plus « crombistes » d'entre nous - la science arabe serait globalement congénère de la science grecque? La scientificité, au sens moderne, de cette dernière, en effet, se limite aux mathématiques, pures et appliquées, les autres domaines étant en quelque sorte caractérisés par un ensemble de techniques parfois fort développées auxquelles sont adjointes des spéculations hyperthéoriques.

L'une des intentions fondamentales de cet ouvrage est de renverser cette idée suggérée par son plan. Dans son article sur "L'optique géométrique », Rashed entend montrer, en effet, que dans le domaine de l'optique - et, dans une certaine mesure, la même thèse peut être soutenue pour l'astronomie —, les savants arabes ont été les auteurs d'une véritable révolution scientifique au sens bachelardien de l'expression. Trois moments fondamentaux scandent l'histoire de l'optique arabe. Ibn Luqa et alKindi posent la nécessité de combiner mathématique et physique, et plus précisément géométrie de la vision et physiologie de la vision. Ibn Sahl, à la fin du IX ${ }^{\mathrm{e}}$ siècle, pousse assez loin l'étude des lentilles pour, entre autres choses, découvrir la loi de Snellius, sept bons siècles avant ce dernier. Enfin, Ibn al-Haytham, combinant, comme personne avant lui, mathématisation et expérimentation, change la nature même de l'optique : il découple l'optique de la vision. «L'optique, écrit Rashed, n'a plus le sens qu'elle revêtait naguère : une géométrie de la perception. Elle comprend désormais deux parties : une théorie de la vision, à laquelle sont également associées une physiologie de l'œil et une psychologie de la perception, et une théorie de la lumière, à laquelle sont liées une optique géométrique et une optique physique » (t. II, p. 310).

Sans doute, certaines des querelles qui ont partagé les spécialistes de l'histoire de la science occidentale pourraient-elles surgir aussi à propos de la science arabe. Où y a-t-il rupture, et rupture de quel ordre? Peut-être pourrions-nous plus facilement 
nous poser ce genre de questions si les auteurs de cet ouvrage avaient trouvé matière à écrire un article sur la dynamique. Ce que les deux articles sur l'optique arabe font ressortir c'est aussi le besoin d'une réévaluation de notre appréciation de l'optique de la fin de l'Antiquité gréco-romaine. C'est en étudiant plus précisément les « expériences » de Ptolémée sur la réfraction, par exemple, que l'on saisira toute la force novatrice d'Ibn-Sahl et d'Ibn al-Haytham.

Ma troisième remarque concerne, elle aussi, l'appréciation globale que l'on peut porter sur la science arabe. Que les Arabes soient les transmetteurs de la science grecque à l'Occident, c'est une idée d'autant plus profondément enracinée dans les esprits qu'elle n'est pas fausse. Mais elle est susceptible de produire les pires contresens. Or l'apport théorique et historique principal de cet ouvrage me semble être la destruction définitive qu'il opère d'une fausse image de la science arabe transmetteuse. Quand l'Occident connaissait Aristote principalement à travers les traductions latines de traductions arabes, directes ou non, du texte grec, c'était mieux que rien. Mais, évidemment, quand le texte grec fut accessible, l'Aristote arabo-latin perdit l'essentiel de son intérêt. Aujourd'hui encore, pour qui cherche à établir les textes grecs, l'intérêt des versions arabes est en quelque sorte accidentel, bien que cet « accident » puisse prendre des proportions gigantesques quand le texte grec est mal établi et que les versions arabes ont été faites sur des manuscrits plus corrects ou quand la traduction arabe seule nous reste alors que le texte grec est perdu. On sait, de ce dernier point de vue, l'importance du «Galien arabe ». Ce modèle, valable pour la transmission des textes philosophiques, a-t-il été indûment étendu à la science arabe? Il semble que oui, et je laisse aux sociologues et historiens le soin de nous dire quelles forces sociales ont poussé les esprits en ce sens. Certes, cette image des Arabes transmetteurs était parfois acceptée juxta modum: on pourrait trouver des historiens des sciences et des techniques, y compris du temps de la colonisation et de l'européo-centrisme triomphants, qui n'ont pas manqué de souligner qu'en bien des points les Arabes ont complété, rectifié et amélioré leur modèle grec. Sans doute, le développement de la recherche apportera-t-il de nouveaux éléments dans ce sens. Ainsi, dans l'article consacré à la technologie, Donald Hill accumulet-il les présomptions d'une invention et d'une transmission à l'Occident par les Arabes de l'horloge mécanique, tout en réservant la décision finale à un moment où les documents auront été mieux exploités.

Si l'Histoire des sciences arabes renverse une image séculaire, c'est parce qu'elle attaque la conception ancienne dans ses œuvres vives, son noyau dur... suivant la métaphore qu'il nous plaira d'adopter, c'est-à-dire dans l'image qu'elle avait des mathématiques arabes. La partie de l'ouvrage consacrée aux mathématiques permet, en effet, d'aborder sous un jour nouveau le problème des rapports entre les deux sciences que sont la mathématique grecque et la mathématique arabe, sciences qui, par leur scientificité même, appartiennent à la même histoire. Car, à l'inverse, ce n'est que verbalement, si l'on peut dire, que la physique d'Aristote appartient à la même histoire que la physique de Newton. La conception ancienne est à peu près la suivante : en géométrie, les Grecs n'ont jamais été dépassés jusqu'à ce que la discipline soit profondément réorganisée à l'époque de Pascal et de Fermat, avant d'explorer des voies nouvelles avec les géométries non euclidiennes, Poncelet, etc. Les Arabes auraient donc été, dans ce cas, des conservateurs, d'ailleurs, de l'aveu unanime des historiens, assez soigneux. En ce qui concerne l'arithmétique et la 
théorie des nombres, les Arabes auraient eu la bonne idée, qui leur avait été comme dictée par leur position géographique et culturelle d'intermédiaires, d'articuler ensemble la spéculation grecque et un système de notation emprunté aux Indiens, les chiffres dits « arabes ». Quant à l'algèbre, dont le nom même aurait dû inciter les historiens à lui chercher des bases arabes solides, voici ce qu'en dit le Grand Larousse encyclopédique de 1960 - qui, il est vrai, ne représente pas le top niveau de l'histoire des sciences de l'époque : «On fait généralement remonter l'origine de l'algèbre au géomètre Diophante d'Alexandrie. L'ouvrage de Diophante [...] appartient plutôt à l'arithmétique. Toutefois, il convient de dire que l'algèbre était connue dans l'Inde à une époque très reculée. Cette science fut introduite en Europe par les Arabes; ce fut à Venise, en 1494, que Lucas Paccioli fit paraître le premier livre d'algèbre publié en Europe. En réalité le véritable créateur de l'algèbre moderne est le Français Viète $[\ldots]$ »

Or on peut dire qu'il ne reste aujourd'hui rien de cette conception « traditionnelle », et que ce changement radical est le fruit de recherches très récentes, et qui sont loin d'être achevées. Travail d'une équipe, certes, mais où le rôle personnel de Rashed ne doit pas être sous-estimé : la disposition typographique de la table des matières de l'ouvrage semble le désigner comme un primus inter pares, mais il est bien plus que cela. Au cours des trente dernières années, Rashed a fait des découvertes de plusieurs types. Certes, qu'un seul individu accumule cette somme de résultats, montre d'abord l'indigence initiale des recherches dans le champ considéré. Mais quand même... Faute de pouvoir faire une véritable typologie des découvertes rashédiennes, j'en donnerai trois exemples. Premier type : les découvertes pures et simples. Au début de son chapitre intitulé « Analyse combinatoire, analyse numérique, analyse diophantienne et théorie des nombres », Rashed y va de son Exegi monumentum : le chapitre en effet porte sur des domaines qu'il a été le premier à distinguer. Deuxième type : l'attribution incontestable d'une fondation. Prenons l'exemple de l'algèbre. Comme tout historien des sciences, Rashed se trouve confronté au problème du commencement d'une discipline. On sait bien que le principal travail de tout novateur consiste à agencer de manière originale des éléments avant lui disjoints; il n'en reste pas moins un novateur. C'est pourquoi les Babyloniens, Euclide et Diophante - et/ou les Indiens, selon le dictionnaire Larousse ne peuvent prétendre ravir à al-Khwarizmi l'invention, au sens propre du terme, de l'algèbre. Non seulement al-Khwarizmi a-t-il, au début du $\mathrm{IX}^{\mathrm{e}}$ siècle, utilisé le premier le terme al-jabr, mais « le but d'al-Khwarizmi est clair, jamais conçu auparavant : élaborer une théorie des équations résolubles par radicaux auxquels peuvent être ramenés indifféremment les problèmes arithmétiques et géométriques, et ainsi pouvoir s'en servir dans le calcul, les échanges commerciaux, les successions, l'arpentage des terres, etc. » (t. II, p. 32). Le paradoxe, signalé par Rashed, de « la simplicité des techniques mathématiques mises en œuvre »si on les compare à celles des Grecs, le caractère embryonnaire de cette algèbre qui ne connaît pas encore les polynômes soulignent en un sens plus fortement la nouveauté de la démarche. Â partir d'al-Khwarizmi se développent des traditions différentes, que Rashed définit comme "arithmétisation » et "géométrisation de l'algèbre », mais qui seraient peut-être mieux décrites comme algébrisation de l'arithmétique et de la géométrie. Il est tout à fait remarquable que les Arithmétiques de Diophante soient traduites sous le titre L'Art de l'algèbre. D'où le troisième type d'innovation intro- 
duit par Rashed dans son étude des mathématiques arabes : l'historicisation de ces mêmes mathématiques. Dans la partie de son chapitre sur l'algèbre qu'il consacre à al-Tusi, Rashed montre bien que cette histoire est une histoire véritable. Il s'agit là d'un symptôme de maturité pour l'histoire d'une science. Ainsi, dans un autre domaine, ce n'est que récemment qu'on a vraiment pris au sérieux le fait qu'il existe bel et bien une histoire de la médecine grecque antique, avec, entre Hippocrate et Paul d'Égine, des retournements, des oppositions, des innovations.

En ce qui concerne la géométrie, l'article de Boris Rosenfeld et Adolf Youschkevitch nous la montre encore très dépendante, à la fois dans les problèmes qu'elle se pose et dans les techniques qu'elle met en œuvre pour les résoudre, du « modèle » grec. Non que les auteurs négligent les innovations des Arabes, mais on a l'impression d'avoir plutôt affaire à une géométrie grecque continuée et complétée par les mathématiciens arabes qu'à une "géométrie arabe ». Un exemple intéressant : en examinant les versions géométriques d'identités algébriques, les auteurs notent qu'al-Sijzi ( $\mathrm{x}^{\mathrm{e}}-\mathrm{XI}^{\mathrm{e}}$ siècles) « a apparemment pensé à généraliser les théorèmes de la géométrie à trois dimensions au cas multidimensionnel » (t. II, p. 160), alors que les Grecs étaient évidemment restreints par leur parti pris géométrique aux trois dimensions de l'espace euclidien. Mais pour de «vrais » algébristes le problème ne change-t-il pas de nature? Peut-être les choses vont-elles bientôt évoluer pour la géométrie elle aussi, et l'originalité de la géométrie arabe s'affirmera-t-elle plus fermement. Quant à l'article de Marie-Thérèse Debarnot, il établit bien l'originalité ce qui ne signifie pas l'absence d'antécédents — de la trigonométrie arabe.

Reste donc un aspect important à considérer pour que les sciences arabes, et principalement les mathématiques, prennent leur place dans l'histoire mondiale des sciences, à savoir l'histoire de leur transmission. Car, quand finit la science arabe, c'est la science européenne qui prend le relais - expression lourde de problèmes, mais qui n'est pas absolument fausse. Le remarquable article d'André Allard sur «L'influence des mathématiques arabes dans l'Occident médiéval » montre à la fois l'ampleur du travail accompli et celle des recherches restant à faire. Exemple : l'impression que l'algèbre a eu du mal, dans l'Europe occidentale médiévale, à s'imposer comme discipline autonome, en se cantonnant au domaine des calculs à but utilitaire, doit-elle être rangée au nombre des vérités historiques? « Fibonacci, écrit Allard, échappe seul à ce jugement sommaire; encore son œuvre ne manifestet-elle qu'un reflet occasionnel d'al-Karaji, d'al-Khayyam ou d'Ibn al-Haytham » (t. II, p. 229).

Il reste donc à faire, et presque tous les contributeurs ont insisté sur ce point dans leurs articles. C'est peut-être aussi l'un des aspects les plus attachants de cette somme, qui avait vocation à dresser un inventaire et un bilan, que de proposer autant de programmes de recherches. 
Amalia BetTini, Cosmo e Apocalisse. Teorie del millennio e storia della terra nell'inghilterra del seicento. Florence, Olschki, 1997. $17 \times 24,322$ p., bibliogr., index (Biblioteca di « Nuncius », vol. XXV).

L'ouvrage comporte deux parties. La seconde est consacrée au débat autour de la cosmologie millénariste qui détermine le lieu et l'époque étudiés : l'Angleterre du $\mathrm{XVII}^{\mathrm{e}}$ siècle, dans laquelle ce mouvement a exercé une "véritable force d'attraction ». La première plante le décor et y place les protagonistes.

Le millénarisme, attente d'un second avènement du Christ, offre l'occasion d'une réflexion sur l'histoire de l'homme et du monde, sur ses possibles scénarios, non seulement passés et tournés vers l'origine, mais encore futurs et orientés vers une eschatologie.

Le millenium se trouve défini dans Apocalypse, XX, 4-6 : à la fin des temps, les élus reviennent à la vie et règnent avec le Christ durant mille ans. Ce lieu de l'Écriture inspirera des mouvements religieux croyant en une Rédemption collective, terrestre, imminente, totale et accompagnée de phénomènes surnaturels.

Dans l'Angleterre du $\mathrm{XvII}^{\mathrm{e}}$ siècle, la question du millénarisme se pose en termes théologiques et métaphysiques. Dans le deuxième chapitre de la première partie et dans le cinquième de la seconde, l'auteur rappelle en outre les origines et les implications politiques de la littérature apocalyptico-eschatologique.

Nous assistons à la succession diachronique de trois millénarismes : le religieux, le politique et le cosmologique. Mais, précise l'auteur, toute succession de cette sorte porte un effet d'illusion rétrospective : les trois moments sont en réalité imbriqués, et cette tripartition ne sert que d'outil interprétatif commode pour un tournant de l'histoire de la pensée politique, philosophique et scientifique moderne.

L'auteur propose de centrer le débat autour de la Telluris Theoria sacra de Thomas Burnet, publiée en 1681. Autour de Burnet, les principaux acteurs, William Whiston, John Ray et Isaac Newton, se rejoignent dans un projet de recherche totale. Connaitre le monde signifie en effet connaître Dieu, puisque les lois qui régissent le monde sont l'œuvre de la toute-puissante volonté de Dieu.

Le premier chapitre présente les premières manifestations de la littérature eschatologique anglaise. La Réforme accentua cette tendance de l'exégèse qui voulait que les prophéties fussent objectives et que l'Ancien Testament préfigurât le Nouveau. La fin des temps voit advenir le règne de Dieu, l'histoire du monde et celle de l'homme étant ordonnées selon un projet providentiel. La prophétie est alors identique à l'histoire, et la Bible, vraie à la lettre, renferme la parole et le secret de ce projet divin. Ce sont les thèses de Newton, exposées à la fin de l'ouvrage.

Une fois posés ces principes philosophiques, la démarche de l'auteur devient plus historique. De Walter Raleigh à Hanserd Knollys, en passant par John Foxe, Thomas Brightman, John Archer, nous voyons se développer le grand courant puritain illustré notamment par John Milton, Samuel Hartlib, Henry More et Isaac Newton, mais aussi par de grands prédicateurs, les «médecins de l'âme » — William Perkins, Richard Baxter — qui, le péché originel ayant fait de tout homme un coupable, préparent la restauration du règne de Dieu sur la terre.

Le deuxième chapitre décrit les divers courants du millénarisme politique. Pour comprendre l'utopie conservatrice du millénarisme modéré, il faut évoquer les lati- 
tudinaires. Selon eux, l'ordre providentiel du monde politique et naturel est l'instrument et le fondement idéologique d'une classe bourgeoise légitimant l'Église et l'État contre la menace des enthousiastes et des utopistes radicaux. Ceux-ci sont à la recherche d'un monde plus juste et égalitaire, caressent le grand rêve de la création d'un nouveau paradis sur terre - selon Gerrard Winstanley, seuls les pauvres sont pressentis comme étant les élus -, de la Societas christiana, du collège universel, lieu d'éclosion de la Pansofia, de Jan Amos Koménsky alias Comenius, du collège invisible de Robert Boyle et John Willis, de la Nouvelle Atlantide de Francis Bacon.

L'auteur, au troisième chapitre, en vient au millénarisme philosophico-théologique de « la vie après la mort ». Quel est le sort de l'âme lors de la résurrection des corps? Pour les «immortalistes »- Walter Charleton, les Platoniciens de Cambridge —, l'immortalité de l'âme est un dogme indémontrable. Pour les thanatopsychistes - Francis Blackburne, Thomas More - l'âme, jusqu'au jour du Jugement, morte au monde, mais vivant en Dieu, repose dans un état de silence semblable au sommeil.

Pour les «mortalistes» - Richard Overton et le poète John Milton —, cette immortalité de l'âme n'est que conditionnelle, ne commençant qu'avec la Résurrection. Matérialisme déguisé? Si l'âme meurt avec le corps ne serait-elle pas toute matière? Le débat est semblable qui agite les panpsychistes: la résurrection des corps et la théorie corpusculaire de la matière sont-elles conciliables?

Dans la seconde partie de l'ouvrage, l'auteur retrace le débat cosmologique qui résulte des thèses précédemment étudiées : qu'en est-il de la création de la terre, de son histoire et de son destin final?

Burnet utilise la physique cartésienne pour tenter d'identifier les causes naturelles qui président aux événements que raconte l'Écriture. Sa cosmologie - dans laquelle la providence divine est cause première, puis spectatrice omnisciente de l'histoire - marque trois étapes : le chaos originel, le déluge et la conflagration finale, promesse d'une nouvelle terre et d'un nouveau ciel. Ces positions vont susciter nombre de polémiques : d'un côté, la reconnaissance d'un mécanisme physique et du bien-fondé de la philosophie naturelle, de l'autre, une critique du monde cartésien où la providence ne joue plus aucun rôle.

Herbert Croft expose les points qui, selon lui, rendent la théorie de Burnet philosophiquement inacceptable : l'usage biaisé du texte sacré; le récit imaginaire du déluge; le fait de considérer la terre actuelle comme étant en décadence. Il convient plutôt de considérer le texte sacré comme intouchable et indiscutable. C'est également le point de vue d'Erasmus Warren qui reproche à Burnet ses graves attaques à l'encontre de l'Écriture. De même, John Keill, du point de vue de la science et de l'astronomie en particulier, dénonce chez Burnet une utilisation abusive du mécanisme.

Les thèses de John Ray ne constituent pas à proprement parler une réponse à Burnet, mais un exposé parallèle de pure physico-théologie : une glorification de Dieu dans Ses œuvres. S'il adopte la «matière immatérielle » de More, Ray semble philosophiquement plus proche de Cudworth en prônant l'action différenciée de la Nature plastique, force vitale interne du cosmos, s'autofaçonnant, inconsciente de son action, instrument et signe de l'omnipotence divine. La méthode de Ray, qui unit l'intention apologétique à l'érudition et à la compétence scientifique, aboutit à 
une pragmatique de la vie sainte, un « manuel de sainteté » qui prépare à la vie éternelle.

L'œuvre de John Woodward pose la question, très débattue dans la perspective d'une « archéologie de la terre », de la nature des fossiles : sont-ils, c'est la position de Woodward, la mémoire de la terre et les témoins de la véridicité de la destruction diluvienne? Ou de simples jeux de la nature, sans autre signification? Les thèses de Woodward sont issues des travaux du Danois Niels Steensen, alias Sténon, qui présentait en 1669 l'hypothèse de la structure stratigraphique de la croûte terrestre et de la nature organique des fossiles. Il démontrait que la dynamique du Déluge n'est pas contraire aux lois du mouvement naturel. Pour Woodward, le récit mosaïque est un document historique, un récit véridique qui décrit des événements scientifiques; n'en pas tenir compte serait tomber dans un impardonnable arbitraire. Mais la pensée de Woodward est potentiellement plus hérétique que celle de Burnet. Dire que le texte mosaïque est scientifique signifie en effet entamer irrémédiablement l'autorité de l'Écriture.

Les travaux de William Whiston, proches de ceux de Burnet et de Ray, développent une problématique élargie : s'y trouvent soulevées la question des comètes et celle des prophéties, que tranchera Newton. L'auteur qualifie d' "occasionnalisme ambigu » l'intéressante position philosophique de Whiston; d'un côté, il semble, avec Newton, soutenir l'intervention continue de Dieu dans le monde, sa providence particulière; de l'autre, il reconnait la nécessité d'une providence générale des lois physiques, se refusant à trancher le nœud gordien du double providentialisme. Sa vision du monde est plus proche de celle de Malebranche que de celle de Descartes.

$\mathrm{Au}$ dernier chapitre, avec Newton, l'auteur manifeste, fort habilement selon nous, dans l'ordonnance même de l'ouvrage, l' «avancée cyclique » propre à l'histoire millénariste : les thèses ici exposées l'étaient au début. Newton comprend la prophétie comme un cryptogramme de l'histoire que seule une intelligence illuminée par la grâce divine peut déchiffrer: la science newtonienne étudie la réalité du monde comme figure de l'ordre divin, mais ce n'est que dans les textes prophétiques, enseignant l'espérance du monde véritable, que Dieu a révélé son projet. Ce projet de recherche totale est bien l'objet du millénarisme religieux.

Amalia Bettini, dans ce riche ouvrage, s'inspire des recherches anglo-saxonnes qui ont souligné l'importance de l'étude des controverses pour l'histoire des sciences, en insistant, à juste titre selon nous, sur leur portée théologique et métaphysique. L'érudition déployée est vaste et les références précieuses pour quiconque s'intéresse à cette période effectivement capitale de l'histoire de la pensée moderne, moment où la conciliation de la raison et de la foi, encore problématique, va permettre le développement d'une science de l'ordre et des lois qui régissent le monde et l'homme. 
Peter Dear, Discipline and experience. The mathematical way in the Scientific Revolution. Chicago/Londres, The University of Chicago Press, 1995. 15 x 22,5, XII-290 p., bibliogr., index.

Les caractéristiques de l'expérimentation et son rôle dans l'avènement de la science moderne sont depuis longtemps des sujets favoris pour les historiens. Dans leur célèbre étude d'une controverse entre Boyle et Hobbes, Leviathan and the air pump, Steven Shapin et Simon Shaffer avaient en 1985 institué comme archétype de l'expérience scientifique la conception qu'ils attribuaient à Boyle et aux premiers membres de la Royal Society et qu'ils reliaient à leur culture intellectuelle et sociale spécifique : l'expérience est une activité instrumentale dont le compte rendu doit expliciter la singularité et les détails concrets, dont le pouvoir de conviction repose en partie sur le statut attribué aux observateurs et dont il s'agit d'abord de multiplier les variantes, sans recours immédiat à une théorie les unifiant, mathématique par exemple.

Peter Dear, tout en reconnaissant l'importance de la problématique de Shapin et Shaffer, souligne d'entrée le particularisme du cas traité, même au sein de la Royal Society. Un projet possible pour étudier l'expérience au XVII siècle (en partie poursuivi par divers historiens depuis 1985) consisterait à explorer d'autres controverses et d'autres groupes savants, en vue d'une typologie plus complète. Dear propose quant à lui une approche transverse, en concentrant son attention sur une pratique discursive commune à de nombreux textes scientifiques de l'époque : cette pratique conçoit d'emblée l'expérience comme productrice d'un énoncé universel. L'objectif du livre de Dear est de montrer comment cette pratique enracinée dans l'enseignement a perduré tout au long du siècle et comment, aux prises avec le changement des relations entre mathématiques, physique et philosophie naturelle, elle intervient dans la transformation des modes de connaissance du monde naturel qui s'opère au cours du XVII siècle.

Un chapitre introductif passe tout d'abord en revue plusieurs notions cruciales expérience, expérimentation, induction - pour préciser leurs acceptions au XVII siècle; dans la lecture usuelle d'Aristote à cette époque, par exemple, l'expérience typique, qui se doit d'indiquer une propriété générale du monde - comment les choses arrivent en général dans la nature et non, comme pour Boyle, quel événement spécifique s'est produit —, est faite de connaissances communes, banales et partagées par tous, qui peuvent ainsi s'utiliser comme prémisses légitimes dans un raisonnement ou un débat.

Les chapitres suivants examinent comment ce point de vue est mis en œuvre dans certains travaux jésuites. Les jésuites, en effet, formateurs importants de l'Europe continentale par leurs collèges ou, indirectement, par la diffusion de leurs textes, apparaissent ici comme des agents exemplaires pour la circulation de cette conception. Mais certains d'entre eux, comme Clavius, sont aussi porteurs d'un projet concernant la hiérarchie des disciplines : selon Dear, un de leurs enjeux est d'établir les mathématiques mixtes — optique, astronomie, musique... - comme de véritables sciences (et donc de réhabiliter les enseignants de mathématiques par rapport à ceux de philosophie). Cela exige, selon les définitions en vigueur, de remplir certains critères; ainsi, les principes du domaine doivent être homogènes au sujet traité, 
un axiome géométrique ne peut par exemple pas servir de principe en astronomie. L'expérimentation peut fournir ces principes, mais le problème se pose alors de légitimer, comme des énoncés universels du sens commun, ce qui résulte d'observations astronomiques accessibles seulement à des experts grâce à un matériel spécialisé, ou de nouveaux phénomènes contestés, ou encore des interprétations controversées. Le livre explore ces différents aspects et les solutions adoptées à travers de nombreux exemples : traités optiques d'Aguilonius et de Scheiner, débats entre Cabeo, Arriaga et Riccioli sur la chute des corps, travail de Grassi sur les comètes. L'analyse est également étendue à des auteurs qui partagent en partie la même exigence discursive, comme Galilée, Mersenne ou Descartes; Dear s'attache à en décrire les variations, en particulier en ce qui concerne le rapport entre expérience et modèle mathématique - pour certains, par exemple, les observations elles-mêmes sont informées, voire corrigées, par la raison théorique.

Le thème des frontières disciplinaires entre mathématiques et physique est ensuite repris pour en souligner le lien avec l'opposition classique entre art et nature : le sens de chacun de ses termes, son évolution et celle de leur relation au cours de la période étudiée sont examinés en fonction de la conception de l'expérience qui s'y trouve privilégiée - représentation mimétique de la nature ou torture exercée sur elle, par exemple.

Les deux derniers chapitres reprennent les exemples classiques des expériences de Pascal sur le vide, d'une part, et de Newton sur le prisme, d'autre part : alors que, dans le premier cas, les récits d'expérimentations singulières qui apparaissent dans sa correspondance sont évacués par Pascal de ses essais récapitulatifs - conformes au mode de discours analysé auparavant —, dans le deuxième cas au contraire, des énoncés généraux conçus dans un cadre mathématique déductif sont reformulés en termes d'expériences concrètes, pour tenir compte des exigences imposées par Boyle et ses contemporains aux publications de la Royal Society. La manière novatrice dont Newton intègre expériences et mathématiques dans le cadre nouveau d'une physico-mathématique expérimentale, abandonnant entre autres l'exigence d'homogénéité des principes, devient pour Dear le modèle de la future pratique scientifique - le point de vue radical de Boyle n'apparaissant finalement que comme une sorte de détour ponctuel.

Cette brève présentation ne rend pas justice à la richesse documentaire et thématique, un des grands atouts du livre, dont témoigne en particulier un alléchant index. L'approche choisie permet des rapprochements suggestifs : on voit ainsi que pour rendre crédible un mode d'expérience au $\mathrm{XVII}^{\mathrm{e}}$ siècle, certains ont été amenés à construire une tradition adéquate pour l'y insérer (p. 115), d'autres ont constitué un groupe de témoins et de garants (p. 79), d'autres encore se sont appuyés sur les recueils de récréations mathématiques (p. 146); dans chaque situation, l'auteur indique les modèles possibles de l'entreprise et les circonstances qui la justifient. De même sur la variation des mots «expérience » et «expérimentation », sur la question des «causes» valant comme explications en sciences, sur l'usage des métaphores, et bien d'autres sujets.

La mise en relation entre des composantes de textes mathématiques et des récits d'expérience ouvre elle aussi des pistes intéressantes : deux exemples convaincants concernent la constitution et le rôle des tables (p. 82, 205), ainsi que le rapproche- 
ment suggéré par l'auteur entre la description opératoire d'une expérimentation et les postulats constructifs en géométrie (p. 212-213).

Mais le plus grand mérite du livre est bien sûr de rouvrir le dossier de la Révolution scientifique et surtout du rapport entre mathématiques et science expérimentale sur de nouvelles bases, informées des critiques issues de la sociologie historique des sciences, mais qui ne s'y réduisent pas. Il ne s'agit ici ni de soumettre, ni d'opposer les mathématiques à l'expérience, mais de scruter une articulation, et surtout de problématiser sa nature et son évolution. Dear donne aussi à voir concrètement certains éléments de continuité avec les traditions scolastiques et montre comment leur intervention contraint, voire motive, des réajustements plus vastes - au niveau des disciplines - jusqu' au point de rupture avec ces traditions. A priori d'ordre strictement intellectuel, ces éléments sont connectés par Dear à des phénomènes culturels plus larges, mais précisément circonscrits.

Ma seule réserve concerne les limites de la description obtenue. Ce que l'auteur argumente de fait touche à l'agrégation entre certaines conceptions de l'expérience et de l'expérimentation et certains projets de refonte disciplinaire, des mathématiques mixtes à la physico-mathématique newtonienne. C'est le niveau exact de cette agrégation qui reste ambigu. Dans l'introduction (p. 5-6), Dear donne l'impression de vouloir décrire un socle commun à la structuration des connaissances, opérant comme une "archéologie du savoir » (la référence à Michel Foucault est d'ailleurs explicite), un niveau qui relève des catégories cognitive et disciplinaire (p. 6) : les modifications à ce niveau traduiraient donc une dynamique profonde des champs concernés et du rapport à la connaissance du monde naturel. Mon objection n'est pas tant celle redoutée par Dear, selon laquelle ce niveau ne serait que langage encore qu'un examen global des différentes pratiques associées, entre autres de la construction et de la mise en œuvre des dispositifs techniques de l'expérience, serait indispensable pour valider complètement la thèse du livre. Le problème me semble surtout résider dans l'assimilation entre «langage» ou plus précisément «discours » (constructeur de signification) et pratique d'écriture textuelle : le lien peut exister, surtout en ce qui concerne les mathématiques, mais il ne va pas de soi et il n'est pas constant (voir, à titre comparatif, pour une période différente, la démarche similaire de Jim Ritter, «Les pratiques de la raison en Mésopotamie », in JeanFrançois Mattéi, éd., La Naissance de la raison en Grèce, Paris, Presses universitaires de France, 1990, p. 99-110). On peut ainsi se demander si ce que Dear repère n'est pas surtout une organisation du traité savant, dont l'impact réel sur les processus cognitifs, ou même les pratiques de recherches, resterait à déterminer. Il ne s'agit nullement de minimiser l'importance et l'intérêt de ce niveau d'études, mais de le situer plus exactement : il n'est pas évident qu'il suffise à repérer le lieu et les forces d'une évolution aussi globale que paraît le souhaiter l'auteur. De fait, les récits d'expérimentation détaillés paraissent réservés à la correspondance (voir Christian Licoppe, La Formation de la pratique scientifique. Le discours de l'expérience en France et en Angleterre, 1630-1820, Paris, La Découverte, 1996) et les échanges autour du Messager céleste de Galilée rapportés dans la belle édition d'Isabelle Pantin (Paris, Les Belles Lettres, 1992 et 1993) témoignent ainsi, dès le début du siècle, de conceptions assez proches de celles attribuées par Dear aux mathématiciens de la seconde moitié. Le changement pourrait tenir à la visibilité nouvelle acquise par ces récits lorsque les lettres sont publiées dans des journaux 
savants; il relèverait donc plus de l'histoire des publications que de celle des disciplines. Les composantes étudiées et leur évolution me semblent donc devoir être scrutées de plus près, d'autant que des travaux récents renouvellent actuellement nos connaissances sur les programmes jésuites et leur influence éventuelle (Luce Giard, éd., Les Jésuites à la Renaissance. Système éducatif et production du savoir, Paris, Presses universitaires de France, 1995, et Antonella Romano, La Contre-Réforme mathématique. Constitution et diffusion d'une culture mathématique jésuite à la Renaissance (1540-1640), Rome, École française de Rome, 1999).

Reste un livre passionnant, par le nombre de pistes nouvelles qu'il propose, son stimulant programme et son effort pour appréhender dans les termes de ses acteurs mêmes des caractères importants de la culture scientifique du XVII ${ }^{\mathrm{e}}$ siècle.

Catherine GoldSTEIN

Anthony Grafton, Les Origines tragiques de l'érudition. Une histoire de la note en bas de page. Trad. de l'anglais (américain) par Pierre-Antoine FABRE. Paris, Seuil, 1998. $14 \times 21,224$ p., index (La Librairie du xx siècle).

Le petit livre, proposé par Anthony Grafton (spécialiste américain de l'érudition à la Renaissance) à partir d'un sujet apparemment anodin et purement technique, la note de bas de page, procède d'un principe d'amplification. Le phénomène érudit de la note de bas de page est en effet présenté comme un symptôme : "La note de bas de page a partie liée, dans le monde moderne, avec l'idéologie et les pratiques techniques d'une profession» (p. 13); «Le surgissement de la note de bas de page distingue l'histoire moderne de la tradition » (p. 28). L'objet du livre va consister à saisir l'avènement de la forme moderne de l'histoire en suivant l'apparition, ou ses étapes progressives, de cette pratique technique de la note, devenue de nos jours extrêmement codifiée, à tel point qu'elle en devient un véritable rite d'initiation et de reconnaissance de la communauté scientifique. Au-delà de cette analyse sociologique, l'auteur en précise les fonctions épistémologiques : "Les notes remplissent surtout deux autres fonctions. D'une part elles persuadent : elles convainquent le lecteur que l'historien a accumulé une quantité suffisante de travail [...]. D'autre part elles indiquent les sources principales que l'historien a réellement utilisées » (p. 27). L'histoire devient une discipline technique, relevant de procédures de vérification et d'évaluation, que l'on a longtemps pensé objectives; elle devient double, examen des sources et construction de récit. La rédaction des notes fait perdre à l'historien l'« innocence de la simplicité narrative» (p. 62) d'un modèle littéraire désormais dépassé.

Après avoir précisé l'enjeu de ce phénomène, la recherche de son avènement va prendre les apparences d'un récit d'enquête. Contrairement aux apparences et aux simplifications, ce n'est pas l'histoire allemande positive du XIX siècle qui aurait imposé cette modernité. Les coupables sont ailleurs, «la piste de Ranke nous reconduit alors plus loin en arrière » (p. 100). L'enquête se fait alors démystificatrice, obligée de remonter dans le passé pour décrire et saisir les progrès de l'instal- 
lation de cette pratique. Le XVIII ${ }^{\mathrm{e}}$ siècle n'apparaît pas non plus comme le moment décisif. Bien que les Lumières aient proposé divers modèles historiographiques, dont Grafton rend bien compte, la pratique de la note n'y apparaît jamais centrale.

C'est plus haut encore que l'enquête aboutit, dans l'analyse de trois époques fondatrices. D'abord l'histoire critique, telle qu'elle se développe au $\mathrm{xvI}^{\mathrm{e}}$ siècle, particulièrement en France, avec François-Auguste de Thou et Étienne Pasquier. Ces juristes, participant à la construction de l'État français, ont été amenés dans ce contexte de fondation à préciser leur rapport au passé, en particulier par leur attention à l'examen des sources de première main. Cet examen est censé établir la vérité et assurer par là la paix civile sans cesse menacée. Cet effort s'est trouvé poursuivi, sans solution de continuité évidente toutefois, par l'histoire ecclésiastique et antiquaire au siècle suivant. C'est ici le nom d'Athanasias Kircher qui doit être retenu, qui consacre quant à lui la publication même des sources en notes, ce à quoi s'était refusé la « génération » précédente. La vérité, là encore, doit être refondée à une période où les connaissances et les voyages remettent en cause les certitudes anciennes. Enfin, «Bayle venait renforcer cette même discipline historique dont beaucoup avait cru qu'il l'avait défiée » (p. 160). La critique baylienne parachève cet ère du soupçon qui agite les connaissances depuis la Renaissance, en puisant, de manière paradoxale, chez Descartes le modèle d'une nouvelle science objective. La double dimension de l'histoire moderne se trouve au fondement même du projet du Dictionnaire historique et critique : définitivement détachée des modèles religieux et strictement narratifs, l'histoire avait désormais trouvé sa méthode.

Ces trois étapes rendent compte, pour Grafton, de la genèse de la pratique de la note en bas de page. Il insiste à de nombreuses reprises, à juste titre, sur le fait que «le fil de la tradition historique suit un cours infiniment plus tortueux et complexe que ces formules ne sauraient le suggérer» (p. 102); «le discours historique en général avance à pas progressif, il ne fait pas de saut; il évolue sans révolution » (p. 172). Il faut souligner combien l'analyse de cette évolution lente, qui voit l'installation de ce locus classicus que sont devenues les notes, met en évidence l'absence d'origine absolue. L'histoire doit ainsi s'attacher à décrire les évolutions, les inflexions et les transformations, bien plus que rechercher à identifier des commencements absolus, qui n'existent pas.

Mais de cette transformation progressive il faut tout de même essayer de rendre compte. Or cette genèse contournée doit beaucoup aux circonstances au sein desquelles la nécessité d'une nouvelle technique apparut indispensable. La Renaissance, la période moderne d'établissement de nouvelles formes étatiques, puis les polémiques religieuses contribuent toutes à rendre problématique le rapport à la temporalité et au passé. Les temps de la pérennité et de la tradition pensés par la religion catholique sont attaqués, remis en cause et critiqués. Cette fragilisation est évoquée par l'auteur, sans qu'il ait toutefois la place de préciser cette contextualisation plus générale, qui aurait peut-être permis de mieux saisir les raisons de cette transformation de l'histoire. Le passé n'est plus désormais ce lent cours d'une tradition assurée par la perpétuation de l'Église; il faut le reconquérir avec des armes nouvelles, dont la note en bas de page apparaît tout à la fois comme l'un des moyens et des symptômes les plus importants. De même peut-on parfois rester sur sa fin lors des analyses, inévitablement concises compte tenu de la taille de l'ouvrage, consacrées aux étapes marquantes, définies comme telles par l'auteur, 
que constituent les œuvres de Thou, de Kircher ou de Bayle. Mais cet ouvrage ne se pose pas comme une somme exhaustive. Il se propose de tracer à grands traits les avènements d'une forme et d'une pratique qui ont présidé à l'apparition de l'histoire moderne. Et, de ce point de vue, le résultat est conforme à ce que l'on pouvait en attendre.

Nicolas PiQué

Laurence Devillairs, Descartes, Leibniz. Les vérités éternelles. Paris, Presses universitaires de France, 1998. 11,5 × 17,5, 135 p., index (Philosophies, 107).

L'objet de l'ouvrage est avant tout de mesurer le rôle architectonique et la fonction médiatrice d'une thèse qui se signale par sa constance et sa cohérence tout au long du développement de la pensée cartésienne.

Son introduction restitue le contenu de cette thèse, énoncée dès les lettres à Mersenne de 1630, qui subordonne les vérités éternelles à la puissance de Dieu. Les précisions que lui apporteront les textes ultérieurs ne la remettront pas en cause. Rien ne peut précéder, dans l'ordre métaphysique, l'existence de Dieu, qui est «la première et la plus éternelle des vérités ». Le vrai dépend de l'existence nécessaire de Dieu, et ne saurait lui être antérieur. Si le vrai annonce pour nous la réalité de quelque chose, il ne saurait précéder logiquement le principe même de la réalité, à savoir l'existence nécessaire ou l'immensité de la nature de Dieu.

Métaphysiques en tant qu'elles se rapportent aux substances pensantes, mathématiques en tant qu'elles se rapportent à l'étendue (voir Entretien avec Burman, part. V, p. 160-161, cité par l'auteur), morales en tant qu'elles déterminent les règles de bonté, les vérités éternelles résident à la fois en Dieu, dans notre entendement où elles sont imprimées, et dans la nature qui y répond.

Mesurer le statut architectonique de la thèse de la création des vérités éternelles revient à s'interroger sur la modalité du passage qu'elle permet d'établir entre la métaphysique et la physique. Selon l'auteur, l'article 36 de la deuxième partie des Principes de la philosophie répond à cette question, en fondant la conservation de la quantité de mouvement sur l'immutabilité divine. La connaissance que nous pouvons avoir de Dieu est le pilier immédiat de la nouvelle physique.

L'incompréhensibilité de Dieu n'est donc pas le constat négatif de la limitation de notre savoir, elle est au contraire l'affirmation positive de ce que nous devons savoir de Dieu pour fonder la science. Comprendre ce que signifie l'incompréhensibilité de Dieu afin d'appuyer sur ce premier savoir l'édifice de la science, telle est la fonction de la thèse de la création des vérités éternelles. Car l'incompréhensibilité de Dieu n'est que le pendant de la parfaite compréhension des vérités éternelles. Si l'être fini comprend les vérités éternelles, il enregistre du même coup que ces vérités, bien qu'éternelles, relèvent d'un monde fini, et leur compréhension ne saurait nous hausser à celle de Dieu infini. L'incompréhensibilité de Dieu est l'affirmation positive de ce que l'idée n'a pas le même statut pour nous et en Dieu. Pour nous, l'idée est (dans le meilleur des cas) la reconnaissance de la vérité : son contenu objectif 
s'adapte à une réalité formelle, celle de la chose, qui lui est extérieure et lui préexiste. Dieu, quant à lui, ne reçoit pas l'idée qu'il se fait d'une chose. Cette idée est en quelque sorte un effet de la volonté par laquelle il la crée. L'incompréhensibilité correspond à l'unité de l'idée et de la volonté, au profit de la volonté. Nous ne pouvons que concevoir la possibilité de cette manière d'être, non la comprendre ni l'effectuer.

L'idée positive que nous avons de Dieu nous impose de reconnaître la différence fondamentale de son mode de connaître, et nous contraint à affirmer que Dieu ne saurait voir sa puissance limitée par ce que nous pouvons concevoir comme impossible. Du fait même qu'elle est compréhensible par nous, la création ne saurait épuiser la puissance divine.

L'indifférence de Dieu qualifie, de manière tout à fait précise, non pas l'extension de la notion de possible à des possibles contraires en Dieu, mais l'abandon pur et simple du concept de possible à propos de Dieu, c'est-à-dire l'abandon d'une distinction entre ce qui est conçu comme possible et ce qui est réalisé par la volonté, donc d'une distinction entre entendement et volonté : elle qualifie l'indistinction (mais non l'identité) de ces puissances en Dieu. De cette indistinction, il suit que la manière humaine de rapporter la liberté à la contingence du choix, et la nécessité à ce qui s'impose d'avance au choix et le limite, ne vaut plus pour Dieu. La puissance infinie de Dieu outrepasse ce qu'il crée comme éternellement vrai et nécessaire : «Définir l'indifférence comme le quomodo de la puissance ne doit pas nous conduire à poser la contingence des vérités éternelles : créées de toute éternité comme vraies et librement comme nécessaires, les vérités ne limitent pas la puissance de Dieu mais ne sont pas pour autant l'effet d'une volonté arbitraire » (introduction, p. 15).

L'auteur insiste sur le caractère définitif de cette indistinction: les vérités, une fois réalisées, ne se haussent pas à l'absolu pour conditionner le choix. C'est maintenant que le nécessaire est libre. Au contraire, «l'infaillibilité du décret divin s'exerce jusqu'aux choses contingentes, qui reçoivent ainsi une immutabilité d'établissement» (p. 16). La contingence des existences est résorbée dans la nécessité avec laquelle toutes les choses sont entièrement soumises aux vérités éternelles.

Que nous fassions l'épreuve de l'inadéquation de nos capacités à la nature de Dieu, ou de l'équivocité des notions qui s'appliquent à Dieu, n'implique en rien que les attributs soient de simples noms de Dieu ou des «manières de parler» (p. 20). L'idée que nous avons de Dieu, comme l'indique la Troisième méditation, est fort claire et fort distincte, et la plus vraie de toutes les idées. Elle est d'ailleurs le fondement de toute certitude, non seulement parce qu'elle garantit l'adéquation de nos notions aux lois du monde, mais surtout parce que l'idée de l'incompréhensible immensité et unité de Dieu nous inscrit, au titre de créature, au sein de la création, c'est-à-dire au sein de la cohérence du système créé. Comme créature, ma nature est l'effet d'un ordre raisonnable, et si Dieu m'a pourvu de pensée, cette pensée est naturellement orientée vers le vrai, parce qu'elle ne peut être orientée vers rien d'autre. L'exercice de mes facultés naturelles convient directement avec ce que Dieu peut faire : «[...] pour être nécessaires, il faut que ces vérités dépendent entièrement de Dieu, que rien ne vienne s'interposer entre son opérer et la présence de ces vérités en notre esprit, pas même la norme de ces vérités, car c'est Dieu même qui l'institue» (p. 22). 
Les deux premières parties de l'ouvrage sont consacrées à l'analyse de la fonction médiatrice de cette thèse. En quoi permet-elle un passage de la métaphysique à la physique? Elle a d'abord une fonction critique. Puisque rien n'est possible en Dieu, la physique ne saurait se fonder sur ce qui nous apparaît comme contradictoire. Ainsi la notion de vide doit être rejetée, car elle affirme le contraire des vérités éternelles en tant que notions primitives : elle suppose une distinction entre l'étendue et la matière. Mais l'infinité de la puissance de Dieu ne s'étend pas à ce que je conçois comme impossible. La confusion de mes idées n'enrichit pas la puissance de Dieu.

Mais le rôle de cette thèse est essentiellement positif : elle permet d'affirmer que les notions par elles-mêmes évidentes pour notre esprit ne sont pas différentes des règles qui régissent les phénomènes naturels. Les lois naturelles, comme établissement, se présentent à tout esprit en tant qu'enseignement, comme règles de sa compréhension du monde. Cette conaturalité, fondée sur l'unicité et la simplicité de l'agir divin, assure le passage épistémologique de la connaissance des esprits à celle du monde. Mais dans la mesure où les notions primitives, qui ne sont autres que les vérités éternelles en tant qu'elles se présentent à mon esprit, m'enseignent que la matière ne diffère pas de l'étendue, le passage qu'elles permettent prescrit également une réduction de toute physique possible à la géométrie.

L'autre apport fonctionnel de la thèse se déduit, selon la deuxième partie de l'ouvrage, de la considération des attributs divins, et tout particulièrement celui de l'immutabilité. Comme l'indique Descartes dans le Monde (XI, 43) : «Quel fondement plus ferme et plus solide pourrait-on trouver pour établir une vérité, encore qu'on voulût la choisir à souhait, que de prendre la fermeté même et l'immutabilité qui est en Dieu? » (cité p. 45). L'article 36 de la deuxième partie des Principes de la philosophie déduit de l'immutabilité divine la conservation de la quantité de mouvement et de repos. L'immutabilité divine apparaît comme la cause première du mouvement. Mais ne limite-t-elle pas l'indifférence de Dieu? En réalité, la constance de Dieu n'est pas une limitation, mais l'effet de son entière perfection. Rien n'est impossible à Dieu, mais il faut reconnaître que l'inconstance serait en lui une imperfection et une impuissance, par suite, il faut affirmer que la volonté de Dieu ne peut pas changer. C'est parce que la volonté de Dieu est immuable que les lois sont immuables, et non l'inverse. L'immutabilité constitue « le point nodal de la thèse, le lieu de la plus haute incompréhensibilité de Dieu » (p. 50), dans la mesure où elle se présente à nous comme une limitation immédiate en Dieu, alors qu'elle n'est que la conséquence de son entière perfection. L'immutabilité comme perfection fonde la constance absolue des lois de la nature. Comme cause immuable du mouvement, elle impose qu'on ne considère dans la nature aucune infirmation des règles. Ainsi les prières des hommes ou les mérites des saints ne sauraient-ils infléchir le cours de la nature. La philosophie naturelle se distingue de la théologie, et les vérités rationnelles prennent le pas sur les vérités révélées. Cette primauté de la métaphysique sur la théologie s'accompagne d'une soumission de la règle de la vie bonne à l'égard des principes métaphysiques de l'institution du monde.

Cependant, l'immutabilité n'implique pas que l'on se débarrasse entièrement des raisons de bonté. La perfection de l'action divine implique sa bonté. Celle-ci nous est inaccessible en elle-même, dans la mesure où « la connaissance des fins de Dieu supposerait que nous puissions comprendre la finalité selon l'unicité de l'entende- 
ment et de la volonté » (p. 68). Que Dieu agisse de façon finale ne nous autorise pas à comprendre l'effet selon une causalité finale. Mais le recours à la finalité n'est en rien exclu. Ainsi, pour défaire l'argument selon lequel l'erreur impliquerait une imperfection dans la création, il faudra bien recourir à des raisonnements dans lesquels entre une mesure précise et contrôlée de finalité (la page 70 et les suivantes présentent un examen très détaillé de la portée des arguments finalistes utilisés dans la Quatrième méditation). L'argument du choix divin et de la perfection du tout impose à l'homme et à la société des hommes la charge et le soin de l'usage correct de facultés en elles-mêmes parfaites (la lettre du 6 octobre 1645 à Élisabeth opère cette extension du raisonnement vers la société des hommes).

La troisième partie de l'ouvrage est consacrée à la critique qu'oppose Leibniz à la conception cartésienne de la création divine. Leibniz propose de rompre avec la thèse cartésienne, qu'il trouve à la fois « trop nécessitariste, faisant du monde créé la mesure de ce qu'il est possible de créer et fondant la bonté du monde sur le simple fait que Dieu l'a créé, et pas assez, faisant des vérités éternelles des produits de la volonté divine en détruisant ainsi l'idée de science divine » (p. 83). Pour Leibniz, le fondement de l'erreur de Descartes réside dans sa conception d'une volonté divine abstraite et sans contenu. Il est impossible de concevoir une volonté qui ne serait pas volonté de quelque chose. Leibniz rejette le principe d'une soumission du monde à une volonté dont on ne pourrait percer les raisons (arbitraires), équivalente à celle d'un tyran (despotique).

Deux conséquences de ce «volontarisme» (p. 83) se révéleraient particulièrement fâcheuses pour Leibniz : d'une part, la possibilité d'une interprétation spinoziste de l'indistinction entre volonté et entendement et, d'autre part, la destruction de la réalité des possibles.

En ce qui concerne le premier point, il est impossible, selon Leibniz, de concevoir comment un pur vouloir, qui ne se veut que lui-même et est absolument nonhétéronome, pourrait parvenir à se déterminer. Avec un pur vouloir, on ne conçoit pas une liberté, car la liberté est autonomie, et l'autonomie suppose non pas qu'on fasse et puis qu'on pense ce qu'on fait, mais qu'on se donne à soi-même la représentation de ce qui est à faire. À la place d'un Dieu libre, Descartes pourrait aussi bien penser un Dieu entièrement nécessaire, car il n'a pas de quoi assurer un véritable concept de la liberté divine. La liberté cartésienne est chimérique, car elle nie la réalité de son objet; au contraire, la liberté consiste à faire toujours ce qu'on préfère raisonnablement, et c'est là le fondement de la félicité et de la gloire de Dieu, fondées sur la notion de personne : la félicité n'est autre que l'amour que l'on se porte en tant qu'être susceptible de bonté. La liberté ne saurait consister dans l'indifférence d'un vouloir vouloir, qui n'est ni une pensée, ni un être. La raison est le contraire de l'indifférence, elle est à la fois la raison qui détermine le jugement et le pouvoir de percevoir cette liaison légitime.

Selon Descartes, de purs possibles sont inconcevables, dans la mesure où la considération du possible et de l'impossible ne saurait valoir pour Dieu, et où de tels possibles ne dépendraient pas de lui. Le pur possible est pour Descartes, comme pour Arnauld, comme aussi pour Spinoza, une pure chimère, fondée sur l'extrapolation de nos manières de voir et sur notre pouvoir de fiction. Or pour Leibniz, le possible doit détenir une forme de réalité, dès lors que l'on perçoit la distinction 
entre les vérités nécessaires et les vérités contingentes. La nécessaire vérité d'une proposition relative à l'existence d'une chose n'est pas identique à celle d'une proposition qui a trait à l'essence de cette même chose. La première est conditionnelle, la seconde absolue. La nécessité absolue (le possible et l'impossible) ne suffit pas pour déterminer un monde à exister. L'existence de ce monde n'est donc pas la réalisation de tout ce qui est possible, mais relève d'un choix entre possibles, qui par conséquent sont dotés d'une réalité indépendante de la production de ce monde.

Que ce monde ne résulte pas de l'épuisement de tout le possible implique directement que les possibles s'ordonnent en séries incompatibles : s'ils formaient une série unique, elle serait immanquablement produite entièrement. L'auteur se range ici à l'avis de Michel Fichant : «La question primitive pour qui demande l'origine des origines n'est pas de dire pourquoi ce monde-là existe (plutôt qu'un autre, ou plutôt que rien), mais comment les premiers possibles répartissent la possibilité de plusieurs mondes différents » (voir «L'origine de la négation », in Science et métaphysique chez Descartes et Leibniz, cité p. 118). C'est sur cette réalité de la distribution des mondes possibles que se fonde la possibilité d'un choix divin et d'un ordonnancement sage du monde. Le possible, même infini, ne se suffit pas à luimême pour être réalisé, car d'autres possibles infinis sont concevables. Le degré de perfection des mondes n'est pas réductible à leur degré de réalité.

La vérité ne se produit pas d'elle-même, par la seule force de sa nécessité, il faut qu'elle soit rapportée à un sujet pensant, et à un Dieu-personne qui, pensant le meilleur monde possible, décide de le créer. Par suite, l'acte créateur n'est pas la simple réalisation ou concrétisation de lois naturelles, mais le choix d'un esprit qui s'effectue par des raisons de bonté. La raison de bonté constitue donc le principe de raison suffisante, mais exprime également le rapport et la société de cet esprit créateur avec les esprits créés. Ainsi, la nécessité des lois relève-t-elle également de ce qui est convenable pour assurer la société des esprits et leur communication, ou leur harmonie. Les idées ou vérités sont à la fois les moyens par lesquels Dieu crée le monde, et des fins qu'il souhaite pour elles-mêmes, et cette réversibilité fonde l'usage des causes finales en physique. Dieu est la fin dernière ou le pourquoi qui répond des phénomènes.

Un monde entièrement réglé par un Dieu qui est sujet et personne, n'implique donc pas seulement la perfection de ses fins, mais surtout la possibilité d'une communication maximale de la félicité de Dieu. Parmi les mondes possibles, Dieu choisit donc celui dans lequel sa communication est davantage assurée. Le critère de la communicabilité constitue la détermination la plus propre de la diversité des mondes possibles, et le critère ultime de leur perfection intrinsèque. Si Dieu sort de lui-même et si la création est un don de Dieu, c'est sa communication qui en est le motif. « Ce monde est choisi à l'exclusion d'autres possibles, car il assure à Dieu la plus grande félicité, incluant celle des créatures. La contingence du monde implique la conception de Dieu comme personne à double titre : en tant qu'il éprouve la plus grande félicité possible, et en tant que ce monde le désigne comme étant ce Dieu » (p. 122).

La connaissance des vérités éternelles est la connaissance de la justice qui règne dans le monde (comme principe du rapport entre esprits), qui est expression de l'amour que Dieu porte aux créatures. La création divine ne s'explique pas totalement par le recours à un Dieu cause des substances. Dieu est avant tout le 
monarque ou le chef des esprits ou des personnes. Ce passage est assurée par la médiation du Messie. Celle-ci est nécessaire pour que les hommes perçoivent la justice de Dieu au sein de la création des substances. «La métaphysique, connaissance des vérités éternelles est donc tout autant théologie, connaissance de l'âme et amour de Dieu» (p. 124). La possibilité d'un accord entre foi et philosophie est ainsi recouvrée.

Philippe DRIEUX

Laurence M. PrinciPe, The Aspiring Adept. Robert Boyle and his alchemical quest. Princeton, NJ, Princeton University Press, 1998. $16 \times 24$, xIv-341 p., bibliogr., index.

Robert Boyle est souvent regardé comme l'un des pères fondateurs de la science moderne. Aussi un livre qui contient des «révélations» sur ses travaux et ses croyances alchimiques flaire-t-il un peu le scandale. Et, de fait, l'auteur met en œuvre toutes les qualités d'un bon détective : l'art de débusquer des sources, de recouper des fragments pour faire des hypothèses, de déchiffrer des codes, etc.

Mais n'attendons pas un livre à sensation animé d'intentions iconoclastes. Laurence Principe s'efforce de retrouver la cohérence de l'œuvre de Boyle grâce à la mise à jour de manuscrits inédits de Boyle (publiés en appendice) et à une relecture mieux contextualisée de ses œuvres publiques. Cet ouvrage participe d'un courant historiographique récent qui conduit à une réévaluation complète du rôle de la chimie dans la révolution scientifique. Nos certitudes tranquilles sur la science moderne, expérimentale, mathématique et mécanique qui chasse l'alchimie avaient déjà été ébranlées par la découverte des manuscrits alchimiques de Newton. Elles sont désormais renversées. Aux travaux de Bernard Joly sur La Rationalité de l'alchimie du XVII siècle (Paris, Vrin, 1992), trois jeunes chercheurs, à la fois érudits et polémistes - William Newman, Antonio Clericuzio et Laurence Principe - ont ajouté, en travaillant de concert, un bel ensemble d'études qui soulignent la continuité entre l'alchimie médiévale et le corpuscularisme des philosophes de la nature du XVII ${ }^{\mathrm{e}}$ siècle.

Le présent ouvrage constitue une pièce maîtresse dans cette entreprise collective de décapage de quelques idées reçues. Premier cliché écarté : Boyle serait l'un des acteurs du passage de l'alchimie à la chimie. D'abord, la distinction terminologique entre alchimie et chimie n'existe que dans l'esprit des historiens. C'est pourquoi Principe choisit d'utiliser les termes en usage au $\mathrm{XVII}^{\mathrm{e}}$ siècle qui faisaient référence aux produits concrets du travail de laboratoire. Il parle de la tradition «chrysopoiètique » (littéralement de «fabrication d'or » ou d' « école mercuriale » par référence à la préparation du mercure philosophique qui constituait une étape vers la pierre philosophale. Deuxième démystification: le Sceptical chymist n'est pas le traité inaugurant la chimie moderne par une critique en règle des doctrines alchimiques. En replaçant l'ouvrage dans le contexte des traditions chimiques qui s'affrontaient $\mathrm{au} \mathrm{XVII}^{\mathrm{e}}$ siècle, Principe montre que les doutes et critiques visent seulement les 
« chimistes vulgaires » que Boyle juge ignorants et parfois charlatans parce qu'il se range du côté des «adeptes », des chimistes véritablement instruits, seuls vrais philosophes.

Loin de rejeter en bloc les traditions chimiques, Boyle condamne les unes en empruntant aux autres. Par exemple, sa critique célèbre des analyses par le feu qui font prendre des «créatures du feu » pour les vrais principes constituants de la nature, se trouvait déjà chez Jean-Baptiste Van Helmont ou Danniel Sennert. Le corpuscularisme que Boyle oppose aux doctrines des trois, quatre ou cinq principes n'est pas un indice de son hostilité aux théories chimiques de la matière. Car ce corpuscularisme a pu trouver son origine dans la tradition chimique, en particulier dans la lecture de Danniel Sennert (William R. Newman, «Boyle's debt to corpuscular alchemy », in Robert Boyle reconsidered, éd. Michael Hunter, Cambridge, Cambridge University Press, 1994, p. 107-118) et il n'implique pas une totale adhésion à une philosophie mécaniste (Antonio Clericuzio, «A redefinition of Boyle's chemistry and corpuscular philosophy », Annals of Science, vol. XLVII, 1990, p. 561-589). Bref, Boyle ne condamne pas la chimie au nom du mécanisme; il prend position dans un débat interne à la tradition chimique. Parmi les « chimistes vulgaires » qu'il critique, Boyle compte les auteurs de cours et de traités de chimie présentés par Hélène Metzger dans Les Doctrines chimiques en France (Paris, 1923) et qu'on regardait, depuis Owen Hannaway (The Chemists and the word. The didactic origins of chemistry, Baltimore, Johns Hopkins University, 1975), comme les agents de disqualification de la tradition hermétique au nom de nouvelles exigences de publicité. Boyle prend donc parti pour les adeptes de l'hermétisme contre l'émergence d'une chimie didactique, ouverte.

L'attitude globale de Boyle à l'égard du secret est le point le plus surprenant de ce livre. Depuis le fameux Leviathan and the air pump de Steven Shapin et Simon Schaffer (Princeton, Princeton University Press, 1985), qui est devenu un classique des études culturelles de la science, on associait l'image de Boyle aux expériences publiques de la Royal Society de Londres qui ont largement contribué à déterminer les conditions de validité de la démonstration expérimentale dans la science moderne. Le Boyle qui organise des mises en scène expérimentales, qui exige des témoins actuels et virtuels, des récits circonstanciés d'expériences ne pouvait, cela va de soi, que condamner les recherches solitaires des alchimistes et leur hermétisme comme une marque d'obscurantisme. Or il n'en est rien. D'une part, le Sceptical chemyst critique l'ambiguité des alchimistes mais il excuse le secret. D'autre part et surtout, Boyle a codé les écrits où il relate ses propres expériences, allant même jusqu'à utiliser neuf codes différents pour voiler ses procédés (p. 143).

Car tout au long de sa carrière, Boyle a lui-même cherché à transmuter les métaux en préparant du mercure philosophique. Son article sur « L'incalescence du mercure avec l'or » publié dans les Philosophical Transactions, 1675-1676, sous les initiales B. R. n'est que la partie émergée d'un iceberg. Non seulement Boyle « croyait » à la transmutation après avoir été témoin de plusieurs expériences, mais il prêtait à l'alchimie une dimension surnaturelle puisqu'il pensait la pierre philosophale comme un relais avec les anges. Boyle était si féru, si enthousiaste, qu'il fut l'adepte zélé - et sans doute la victime — d'une obscure société française d' "Astérisme », sur laquelle pèsent les plus gros soupçons de charlatanisme. 
Voila donc le portrait héroïque d'un père fondateur de la science moderne sérieusement révisé. Mais le grand mérite de ce livre est de ne pas se contenter de renverser une idole. Il parvient à dépasser ces contradictions apparentes, à redonner consistance à un personnage polymorphe en soulignant le rôle central de la religion et du mystère dans la philosophie naturelle de Boyle.

Ce «nouveau Boyle » n'est pas un solitaire. Sa quête de la pierre philosophale le place au cœur d'un collectif désigné comme « école mercurialiste ». Boyle entretenait des correspondances avec des adeptes par toute l'Europe, il travaillait en collaboration avec des «laboratoires satellites », et, à la veille de sa mort, il passa le flambeau à Locke et à Newton. Ce réseau international d'alchimistes apparaît si intimement enchevêtré à la Royal Society non seulement par ses membres mais aussi par ses idéaux et ses aspirations qu'il est désormais impossible de séparer la quête alchimique de la naissance de la science moderne.

Parce qu'il révèle l'ampleur des préjugés qui ont façonné et biaisé les récits des débuts de la science moderne, cet ouvrage devrait intéresser, voire interpeller, tous les historiens quelle que soit leur spécialité, quelle que soit leur époque.

Bernadette BENSAUde-VINCENT

Guillaume Lamy, Discours anatomiques. Explication méchanique et physique des fonctions de l'âme sensitive. Éd. par Anna Minerbi Belgrado. Paris/Oxford, Universitas/Voltaire Foundation, 1996. 17,5 × 24, 192 p., bibliogr., index (Libre pensée et littérature clandestine, 3).

La collection «Libre pensée et littérature clandestine » se propose de faire découvrir ce que l'on peut appeler la «face cachée des Lumières », pour reprendre une expression paradoxale dont Miguel Benitez a fait le titre d'une riche étude sur la littérature clandestine, publiée dans cette même collection, en éditant des textes qui n'avaient jamais connu les faveurs d'une édition moderne ni les grâces d'une étude universitaire.

À l'ombre des quelques grands systèmes de la pensée classique se développe une littérature décidément rebelle à toute tentative de classification, tant par la diversité de ses thèmes, religieux, scientifiques, politiques, philosophiques, que par sa forme, ses procédés argumentatifs, et, il faut bien le dire, sa teneur conceptuelle (n'est pas Descartes ou Spinoza qui veut). Peut-être alors le seul trait — à défaut de genre commun à cette littérature, serait-il un certain style : celui dans lequel s'exprime une liberté, dans son opposition parfois rageuse plus que lucidement critique à toute espèce d'orthodoxie. Comme le négatif, cette littérature travaille la cohérence des pensées toutes faites, et les oblige à constamment se refaire : d'où son importance, en dépit d'une apparence parfois négligeable, et son rôle majeur pour le développement de la pensée de la Renaissance aux Lumières.

Parus en 1675, en même temps donc que le deuxième tome de la Recherche de la vérité de Malebranche, deux ans avant l'Éthique de Spinoza, les Discours anatomiques de Guillaume Lamy exposent les grandes lignes d'une anatomie informée - 
Anna Minerbi Belgrado le montre - par les théories scientifiques contemporaines, en même temps qu'ils concentrent les thèmes classiques d'une philosophie matérialiste et athée, Lamy combinant de manière inégalement heureuse les sources où il puise : Épicure, Gassendi, Descartes. À noter principalement parmi ces thèmes celui bien connu de l'opposition à l'anthropocentrisme et au finalisme. Mais le rejet des causes finales, jugées « inutiles» et «incertaines », prend ici dans le contexte de l'anatomie un relief particulier et plus original : Lamy, s'opposant de manière tranchée aux galénistes sur la question du rapport organe/fonction, soutient qu'il y a dans le corps des parties sans usage (par exemple, le péricarde dont l'ablation n'est suivie sur un chien d'aucun effet visible).

La fin des Discours anatomiques puis l'Explication méchanique élargissent la perspective en donnant une interprétation matérialiste de l'âme, après avoir rapidement réfuté le dualisme à l'aide d'arguments peu originaux, pour l'essentiel tirés des Objections de Gassendi aux Méditations de Descartes. Lamy distingue pourtant, ou feint de distinguer de l'âme sensitive, objet de son propos, une âme raisonnable, dont il assure ne pas nier l'existence. Mais cette âme raisonnable n'est bien sûr connue que par la foi. L'alibi fidéiste, brandi pour la circonstance, laisse peu de doutes sur le véritable sens du propos de l'auteur...

Refusant le dualisme, amené donc à expliquer la sensation par le simple mouvement de la matière, Lamy se verrait contraint d'admettre, paradoxe auquel s'expose tout matérialisme, que la matière pense, et donc puisque tout est matière, que tout, d'une certaine manière, pense ! Or il est particulièrement manifeste chez Lamy, que c'est dans l'effort pour surmonter cette contradiction, bien plus que dans ses faibles arguments contre le dualisme, qu'il se montre le plus audacieux, et que son opposition au cartésianisme porte vraiment. Lamy devra montrer en effet (Explication méchanique, $1^{\text {re }}$ partie, chap. viII), que la matière, quoique généralement étendue, impénétrable et mobile, «n'est pourtant pas entièrement la même ». C'est rompre avec la thèse cartésienne de l'homogénéité d'une matière indifférenciée, c'est admettre des qualités particulières à certains corps. En cela on peut croire que Lamy retourne à des positions dont la critique cartésienne avait démontré l'insuffisance. Il n'en est rien, et c'est même là que Lamy se montre le plus moderne et le plus intéressant. Il est en effet de ceux qui travaillent pour substituer à la définition de la matière par l'extension - définition pauvre, puisque la matière ainsi définie n'est rien d'autre que la pure quantité géométrique (Descartes, Principes, II, art. 64, materia, vel quantitas), et manque si l'on peut dire d'étoffe, de matérialité — une définition plus riche d'une matière, dont les transformations ne se laissent pas entièrement réduire aux lois du mécanisme. Telles les transformations chimiques, dont Lamy soutient contre Descartes la spécificité et l'irréductibilité. Ainsi, avec par exemple du Hamel ou Thomas Willis (que, selon A. Minerbi Belgrado, Lamy utilise constamment sans jamais le nommer), Lamy œuvre pour dépasser le géométrisme cartésien, au moment où, déjà, la simplicité révolutionnaire et prodigieuse de l'étendue modifiée par figure et mouvement semble avoir perdu de sa fécondité, et semble même fonctionner comme frein, voire comme obstacle épistémologique.

Edouard MeHL 
La Matière et l'homme dans l'Encyclopédie. Actes du colloque de Joinville (1012 juillet 1995), textes recueillis par Sylviane Albertan Coppola et AnneMarie ChoullLet. Paris, Klincksieck, 1998. $16 \times 24,345$ p. (Actes et colloques, 49).

Une large part de l'Encyclopédie de Diderot et D'Alembert est consacrée à la matière sous toutes ces formes. La société Diderot s'est donné pour objet d'étude « la matière et l'homme » en mobilisant vingt et un intervenants qui traitent des différents aspects de cette question centrale.

Une première partie concerne la philosophie de la matière. Marian Skrzyek étudie les notions de «matière universelle » et de « grand tout» dans les articles que Diderot consacre aux philosophes anciens puis dans ses propres travaux, preuve d'une continuité trop souvent sous-estimée. Hisayasu Nakagawa aborde le problème du rapport entre l'âme et le corps, la pensée et l'étendue chez Diderot en accordant une place essentielle au principe de la "sensibilité » comme «caractéristique générale de la matière ». Sylviane Albertan-Coppola souligne le caractère fondamental de l'article «Animal », jalon essentiel dans l'itinéraire philosophique de Diderot, mais qui marque également un temps fort dans l'élaboration d'une riposte chrétienne. Dès 1758, l'abbé de Saint-Cyr note qu'en présentant le sentiment comme une propriété physique de la matière, Diderot induit que la pensée en est aussi un attribut. A. J. Chaumeix montre que de l'attribution de la pensée à la matière à la négation de l'âme, il n'y a qu'un pas que le philosophe franchit allègrement. Le P. Hayer et J. Soret, dans La Religion vengée (1757-1763) dénoncent une pensée déterministe en commentant, non sans lucidité les articles « Animal », «Encyclopédie » et «Évidence ». L'article «Animal» a finalement peu de chose à voir avec la zoologie, c'est «Straton, Spinoza et Locke réunis ». Avec l'article «Beau», Georges Filloux développe la notion de perception des rapports dans l'articulation de la théorie matérialiste de la connaissance. Éliane Martin-Haag s'intéresse à une source qui réfute le matérialisme athée, Le Vrai Système intellectuel de l'univers (1678) de Ralph Cudworth, et montre l'influence de cet ouvrage sur Diderot, en particulier dans les Pensées sur l'interprétation de la nature (1753-1754).

Après ces approches philosophiques globales, six contributions sur le thème « la matière et la vie » envisagent les conséquences du matérialisme sur des secteurs plus précis. Marie-Christine Maurel célèbre, avec le passage de la matière inerte à la matière vivante, les débuts de l'approche scientifique du monde : «La vie résulte du dynamisme propre à la matière. Toute explication par Dieu est inutile [...] Le matérialisme "dynamique" de Diderot contribue à évacuer l'aspect théologique de la création [...]» Et l'auteur de rappeler la célèbre formule de Diderot, qui, non sans humour en ce qui le concerne, proclame : "Voyez-vous cet œuf? C'est avec cela qu'on renverse toutes les écoles de théologie, et tous les temples de la terre [...]» Marie-Jeanne Boisacq analyse l'article «Anatomie » et montre le grand intérêt de Diderot pour cette science expérimentale : il défend son statut scientifique et réclame son émancipation de la théologie. Tout comme l'ouvrier horloger est à même de réparer les horloges parce qu'il connaît leur fonctionnement interne, le médecin doit connaître la machine humaine : «L'anatomie devient pratique d'artisan, de mécanicien préposé à l'entretien de la machine [...] » Poussant à l'extrême limite un rai- 
sonnement swiftien, Diderot émet le vœu, heureusement sans être suivi, du moins on peut l'espérer, que les dissections ne soient plus pratiquées sur des cadavres mais sur des criminels. La mort du méchant est plus utile dans un amphithéâtre que sur un échafaud. Soucieux de ménager la sensibilité de tous, Diderot propose que l'on accorde la vie au disséqué s'il survit à l'opération. Lucette Perol étudie une constellation d'articles autour de l'article «Chair » qui promène le lecteur de l'étal du boucher à la métaphysique. À travers cette notion, nous saisissons véritablement l'étendue du projet philosophique, sa cohérence comme les impondérables de sa réalisation. Nous sommes ainsi amenés à réfléchir à l'organisation matérielle du commerce, aux sciences de la vie, aux tabous alimentaires et, bien entendu, à la théologie. Contrairement à ce que l'on pourrait penser, les articles métaphysiques ne donnent pas lieu à de vigoureuses critiques; l'absence de renvoi à l'article « Âme» permet de mieux comprendre cette apparente modération. Le mot «chair» apparaît au début du tome III à l'issue de la plus grave crise traversée par l'entreprise encyclopédique, la prudence s'impose donc. Béatrice Fink se réfère aux textes et aux planches de l'Encyclopédie pour étudier les «matières alimentaires en mouvement» au travers de deux axes, l'assimilation de la nourriture par le corps qui rejoint le paramédical, et les chaînes de production alimentaires, du blé au pain, du sucre aux confiseries. L'article «Chocolat» rédigé par Diderot qualifie cette délicate boisson de « lait des vieillards » et dresse « la belle trinité du bon, du vrai, du savoureux ». Servanne Woodward cerne l'article «Lait» en relation avec les planches «Laiterie » et « Fromage». Elle conclut à « une logique douteuse du lait dans l'Encyclopédie » qui tient sur cette curieuse matière des discours pour le moins contradictoires, l'équivalence des laits est affirmée au point de préférer que tout enfant humain soit nourri aux dépens des animaux plutôt que des nourrices humaines; mais, d'autre part, les laits matriciel et mammaire sont identifiés au point d'affirmer que seul le lait maternel convient à l'enfant... Alain Cernuschi s'intéresse au passage de « la matière sonore » à la musique, à partir de deux sondages dans les discussions sur la validité de la théorie harmonique de Jean-Philippe Rameau et dans les réflexions sur les effets thérapeutiques de la musique. Avec l'article «Harmonie », Rousseau repense le moment où l'harmonie naturelle s'inscrit dans une durée musicale à travers une analogie avec le langage: «Un dictionnaire de mots élégants n'est pas une harangue, ni un recueil d'accords harmonieux une pièce de musique. Il faut un sens, il faut de la liaison dans la musique comme dans le langage; mais où prendra-t-on tout cela, si ce n'est dans les idées mêmes que le sujet doit fournir?»

Les quatre contributions suivantes relèvent de la culture matérielle. Mauricio Jalon étudie la traduction en espagnol de l'Encyclopédie méthodique (en 1794) et note l'attention particulière portée aux aspects techniques et industriels. Franck Salaün rappelle combien les définitions de la matière se heurtent « au système axiologique catholique et au statut ontologique et moral de la civilisation matérielle ». Face aux oppositions corps/esprit, forme/matière, l'Encyclopédie qui relie les formes matérielles et intellectuelles de la culture «court le risque d'être accusée d'idolâtrer la matière ». Gabriel Gohau s'intéresse à la terre dans l'Encyclopédie, c'est le baron d'Holbach qui a rédigé les articles «Charbon », "Volcan », «Fossiles », «Minéraux »..., ses contributions sont comparées à l'œuvre de Buffon. Madeleine Pinault Sorensen reprend les quatre éléments ou corps primitifs, l'air, le feu, l'eau, la terre, et analyse leur place dans l'Encyclopédie. Ils sont liés à l'intro- 
duction du machinisme comme à la sensibilité ambiante et à l'attrait pour le spectacle de la nature.

Une dernière partie consacrée au travail de la matière envisage des applications plus techniques. Antoine Picon s'interroge sur la classification des arts et métiers, classification qui s'opère curieusement en fonction des matières. L'auteur y voit une illustration de la réflexion des Lumières sur le toucher et le geste et l'indice d'une évolution qui va donner naissance à la notion moderne de travail. Paolo Quintili s'intéresse à l'article «Industrie » de Quesnay-Jaucourt. La révolution industrielle n'est qu'à ses débuts, mais les contributions techniques du dictionnaire prouvent qu'une certaine conscience du phénomène se fait jour. C'est le cas notamment lorsque l'on envisage la création de "métamachines », des machines qui fabriquent des machines et qui travaillant à la place des hommes allègent leurs efforts; nous sommes déjà dans le rêve de l'industrie mécanisée. Hélène Vérin s'interroge sur la place des ingénieurs et le rôle que l'Encyclopédie leur confère : "Toute l'histoire des ingénieurs, depuis que l'on en parle et qu'ils parlent de leur art peut se comprendre comme un assujettissement du principe d'une chronologie des tâches ouvrières réglées par les états de la matière et guidées par l'expérience de ce que l'on a déjà fait, au principe hypothético-déductif qui détermine et ordonnance des opérations selon des anticipations guidées par les mathématiques et l'expérimentation physique. » Élisabeth Lavezzi raconte comment Diderot avec l'article «Émail » présente en les dramatisant les difficultés de cette peinture. Nicole Fierobe remarque enfin la précision de l'observation et la passion terminologique qui permettent aux articles consacrés au verre et à la verrerie de contribuer aux progrès scientifiques de la chimie et de la mécanique. Une promenade intellectuelle parmi les forges de Buffon à Montbard, avec comme guide Christian Albertan, clôt ce volume en illustrant un des rêves des Lumières, une «philosophie industrielle».

Monique CoTtRet

Martine Groult, D'Alembert et la mécanique de la vérité dans l'Encyclopédie. Paris, Champion, 1999. 16,5 × 24, 506 p. (Les Dix-Huitièmes Siècles, 34).

Dans son étude sur D’Alembert, Martine Groult entend délaisser délibérément le personnage du mathématicien et du savant pour ne s'intéresser qu'à celui de l'encyclopédiste et du collaborateur de Diderot. Partant du principe, rarement admis par les commentateurs, qu'il existe entre les deux éditeurs de l'Encyclopédie une " profonde entente philosophique » (p. 13), son projet est de montrer comment D'Alembert, à travers sa participation à l'entreprise encyclopédique, a constamment cherché à mettre au jour la «mécanique de l'entendement », la « marche de l'esprit » (p. 13) qui est à l'origine de la découverte de la vérité et de l'invention scientifique en général.

Avant d'entrer dans le vif de son sujet, M. Groult expose brièvement quelques considérations purement biographiques. Après une évocation rapide de la jeunesse de D'Alembert au collège des Quatre-Nations, la première partie de l'ouvrage 
dresse l'inventaire de ses principales publications scientifiques et de ses diverses contributions philosophiques et littéraires. Cette mise au point chronologique comprend encore un rappel de ses relations avec Julie de Lespinasse et quelques brèves remarques concernant l'attitude morale du philosophe.

Dans la deuxième partie de son livre, $\mathrm{M}$. Groult cherche à établir l'originalité du projet encyclopédique, tel que l'ont conçu D'Alembert et Diderot, en opposant celui-ci à «d'autres expositions du système des connaissances qui ont revêtu la même forme de dictionnaire encyclopédique au $\mathrm{xVIII}^{\mathrm{e}}$ siècle» (p. 14). Cette démarche la conduit tout d'abord à examiner la relation de l'Encyclopédie avec la Cyclopedia de Chambers. Des analyses de M. Groult, il ressort que le point de départ imposé par les libraires - la traduction de la Cyclopedia - a constitué pour les deux éditeurs un «poids à traîner » bien plus qu'un « véritable point d'appui » (p. 119). L'introduction des articles consacrés aux techniques, le caractère raisonné du dictionnaire, la dimension collective de l'œuvre opposent profondément l'ouvrage dirigé par D'Alembert et Diderot à celui de Chambers. M. Groult montre encore comment les quatre volumes de Suppléments édités par Panckoucke de 1776 à 1777 , dans lesquels le principe des renvois a disparu, s'écartent du projet initial des éditeurs : "Tout dans le Supplément est sous le règne de l'amalgame et rien n'est relié à quoi que ce soit » (p. 97). Cet écart, enfin, est encore plus sensible en ce qui concerne l'Encyclopédie méthodique publiée à partir de 1782. La «Méthodique », dans laquelle l'ordre des matières remplace l'ordre alphabétique et les renvois du Dictionnaire raisonné, consacre selon M. Groult «la dispersion de la science en matières scientifiques »(p.107). Le public visé n'est plus le lecteur éclairé auquel s'adressaient D'Alembert et Diderot, mais un cercle étroit de spécialistes et de professionnels.

La troisième partie de l'ouvrage est consacrée à l'étude de la «mécanique de la vérité » proprement dite. M. Groult cherche à dégager les idées de D'Alembert concernant l'invention et la méthode scientifiques en étudiant ses positions à l'égard des quatre «génies » considérés dans le Discours préliminaire de l'Encyclopédie : Bacon, Descartes, Pascal et Newton. De Bacon, D'Alembert retient l'exigence d'une présentation du savoir qui ne soit pas un simple catalogue, mais une expression des progrès de l'esprit manifestant un ordre raisonné des découvertes. L'influence de la pensée cartésienne sur le projet encyclopédique est aux yeux de M. Groult la plus déterminante. Comme l'auteur du Discours de la méthode, auquel il voue une admiration indéfectible (ses critiques ne visant jamais que la pensée figée des « sectateurs » de Descartes et non le philosophe lui-même, ni ses « successeurs » authentiques), D'Alembert ne s'impose pas seulement de «faire de la science », mais aussi de montrer « comment se fait la science » (p. 156), c'est-à-dire de mettre en évidence «le chemin par lequel l'esprit parvient à la découverte » (p. 155). L'exemple de Pascal, qui fut à la fois homme de lettres et géomètre, semble quant à lui avoir inspiré l'idéal dalembertien d'un esprit universel (l'esprit « géométrique » par opposition à l'esprit seulement « géomètre » borné à la pratique des mathématiques). Avec Newton, enfin, D’Alembert trouve le modèle d'une physique qui s'en tient « aux observations et aux calculs » (p. 152). Cependant, son ralliement à la science newtonienne ne se fait pas sans réserves : à l'auteur des Principia, il reproche, selon M. Groult, d'avoir dissimulé sa méthode. Son projet serait en quelque sorte « de faire le contraire de Newton, c'est-à-dire de ne pas cacher ses 
efforts, de montrer son esprit au travail » (p. 279). M. Groult montre ainsi comment l'explicitation de la méthode suivie par les «génies inventeurs » conduit D'Alembert à l'idée d'une "métaphysique des sciences » mettant en évidence l'ensemble des opérations «qui déterminent la marche de l'esprit dans la science» (p. 243). Cette exigence de méthode, qui se trouve exemplairement réalisée dans l'analyse des corps que propose la physique expérimentale, ne se borne cependant pas à la connaissance de la nature: M. Groult termine son étude en examinant comment, chez D'Alembert, les notions fondamentales d' « esprit philosophique » et d' « esprit systématique » s'appliquent également au domaine du sentiment esthétique.

Le livre de M. Groult vient confirmer, à la suite des travaux récents de Véronique Le Ru - Jean Le Rond D'Alembert philosophe, Paris, Vrin, 1994 - et de Michel Paty - D'Alembert, ou la raison physico-mathématique à l'âge des Lumières, Paris, Les Belles Lettres, 1998 — le renouveau des études dalembertiennes. On trouvera en effet dans ce travail des vues nouvelles et de nombreuses informations concernant le contexte intellectuel dans lequel s'inscrivent l'entreprise encyclopédique et la pensée philosophique de D'Alembert. Signalons, enfin, que l'ouvrage a le mérite de présenter en annexe une liste exhaustive des articles de D'Alembert dans l'Encyclopédie et dans le Supplément de 1776-1777, ainsi qu'un inventaire de ses articles de synonymes.

Alain FIRODE

Louis Jurine, chirurgien et naturaliste (1751-1819). Éd. René SigRIST, Vincent BARRas et Marc RatclifF. Chêne-Bourg (Suisse), Éditions Médecine et hygiène Département livre Georg, 1999. 17 × 24,5, 495 p., fig., index (Bibliothèque d'histoire des sciences, 2).

L'institut Louis Jeantet de Genève offre par ce nouveau volume de la collection "Bibliothèque d'histoire des sciences ", au-delà d'une belle édition illustrée de planches originales en couleurs, le portrait d'un chirurgien et naturaliste oublié. À travers la figure de Louis Jurine, les éditeurs René Sigrist, Vincent Barras et Marc Ratcliff dirigent une enquête qui se veut la redécouverte d'un homme et d'une œuvre. Tout en décrivant la pratique du chirurgien, qui lui octroie statut social et revenus, le souci de définir la filiation intellectuelle d'un travail scientifique, envisagé dans une Europe des savoirs naturalistes, anime les auteurs qui répondent collectivement à un projet d'histoire : la réhabilitation du genre biographique.

L'ouvrage est introduit par deux articles qui retracent « les étapes d'une double carrière » : Sigrist reconstruit très habilement, en dépit d'archives minces ou dispersées, la pratique chirurgicale et la passion naturaliste de Jurine. Suit un diptyque sur l'œuvre du Genevois : d'une part, une analyse des publications ou des collections patiemment amassées; d'autre part, des réflexions sur les travaux inédits lus par les contemporains du chirurgien. En fin d'ouvrage, des textes originaux de Jurine apportent matière au lecteur qui souhaite prolonger la réflexion.

Étrange Louis Jurine tombé dans l'oubli à sa mort aussi vite qu'il était devenu célèbre... Sigrist ouvre le ban de l'ouvrage en retraçant l'itinéraire du chercheur 
autodidacte genevois, qui cultive les paradoxes. D'origine modeste, sa notoriété est européenne sous l'Empire : cas exceptionnel dans une communauté de savants non professionnels à qui l'aisance financière permet l'étude. Considéré par ses pairs comme un chercheur de premier plan, Jurine n'a pas eu de véritable formation scientifique. Habile chirurgien, il deviendra un médecin reconnu grâce à son mémoire sur le croup, sanctionné par le premier prix du concours impérial. Aucune part de l'activité de Jurine n'est oubliée : l'homme dans son entier - le chirurgien, le naturaliste, le père attentionné, le citoyen - est restitué au travers des correspondances, des archives d'institutions scientifiques et de villes. Le texte, troublant de richesse et d'honnêteté, plonge le lecteur, avec une inquiétante facilité, dans une vie qui s'accomplit.

Dans un même souci de rigueur, le second article de Sigrist nous propose, dans l'Histoire des monocles (posthume, 1820) et dans d'autres travaux publiés ou inédits de Jurine, une lecture fouillée de la démarche scientifique et de la pensée de l'entomologue genevois. L'historien ne manque pas de repérer la démarche d'anatomiste qui prévaut chez le chirurgien, à travers trois éléments : l'importance de la dissection, qui permet de constituer avec succès une ostéologie et une myologie descriptives, mais aussi fonctionnelles; l'usage efficace du dessin pour présenter les résultats d'observations et le recours à l'analogie « pédagogique » des corps d'insectes avec celui de l'homme, grâce à la terminologie souple du chirurgien. Cette imperceptible métaphore filée de l'insecte-homme se poursuit dans le style narratif, proche de celui des romanciers, qu'utilise Jurine pour exposer les mœurs des monocles. Plus avant, Sigrist explore la démarche expérimentale où l'expérience est postérieure à l'accumulation d'observations, comme subordonnée à la mise à l'épreuve de théories explicatives. Celle-ci prend sens dans une «stratégie d'enquête» scientifique consciente de sa force et de ses limites. Le lecteur entre ainsi en profondeur et avec délectation dans la démarche d'un homme qui entend s'inscrire dans une histoire collective et progressive de l'entomologie et dans une pensée qui cherche à faire partager son adoration pour cette «main qui a crayonné également l'homme et la mouche », expression que Jurine emprunte à Charles Bonnet.

Cette démarche est complétée par la mise en perspective de la sémantique de l'histoire naturelle qu'effectue Ratcliff, permettant de montrer, sur un XVIII ${ }^{\mathrm{e}}$ siècle qui s'achève avec Jurine, la réalité de l'évolution de la langue scientifique naturelle, et conteste l'idée défendue par Michel Foucault, selon laquelle la langue des naturalistes serait «massivement morphologique».

En consacrant un article entier au cabinet de Jurine aujourd'hui largement disparu, René Sigrist et Éric Asselborn, replacent la pratique du collectionneur au centre de l'activité naturaliste. En effet, le cabinet de Jurine est scrupuleusement décrit, à la lumière de certains choix de classifications - en entomologie notamment - qui, par leur limpidité, visent à favoriser la formation des naturalistes. L'analyse détaillée des différentes collections, de leurs usages et de leur devenir constitue un document précieux pour la compréhension du rôle de ces objets dans la constitution des savoirs naturalistes. Même si l'on regrette que les auteurs ne soient pas allés plus loin dans l'analyse épistémologique des relations entre les objets des collections et la production de connaissances naturalistes.

«Les dilemmes du systématicien » permettent d'approfondir l'analyse du cabinet. René Sigrist et Patrick Bungener y dévoilent les réactions de Jurine face à dif- 
férentes classifications. Le naturaliste refuse le système de Fabricius fondé sur l'examen des parties de la bouche, difficile à mener aisément et correctement. Aussi plaide-t-il pour des critères qui allient facilité de reconnaissance, invariabilité et unicité, ainsi que valeur pédagogique. C'est dans une approche foucaldienne, qui oppose système et méthode, que les auteurs tentent de situer la pensée du collectionneur naturaliste. Cependant, il ne faudrait pas se méprendre sur ce « Jurine, sorte de pionnier de la systématique dans le microcosme genevois, [qui] en illustre également les impasses dans le cadre du paradigme bonnetien $»$. Les naturalistes des $\mathrm{XVIII}^{\mathrm{e}}$ et $\mathrm{XIX}^{\mathrm{e}}$ siècles éprouvent les difficultés des classifications communes aux tentatives d'ordonnancement du savoir. Du Prospectus de l'Encyclopédie qui préconise le recours au «système des connaissances humaines » de Bacon, à la philosophie allemande $\mathrm{du} \mathrm{XIX}^{\mathrm{e}}$ siècle, en passant par le Traité des systèmes de Condillac, le dilemme entre catégories artificielles et catégories réelles ou naturelles est permanent au XVIII ${ }^{\mathrm{e}}$ siècle. Les naturalistes sont au centre de ces débats, par le maniement du concept d'espèce et des catégories systématiques qui sont problématiques. Penser Jurine hors de ce champ épistémologique ne peut conduire à en faire un pionnier; l'isoler dans le paradigme bonnetien trahit un nationalisme conceptuel suisse qui n'a que peu de sens dans les sciences naturelles du début du XIX ${ }^{e}$ siècle.

Après ces lectures convaincantes, on se demande pourquoi l'Histoire des poissons du lac Léman est exemplaire dans ce qu'elle fait appel aux connaissances des pêcheurs - pratique qui remonte à tout le moins aux travaux de Gesner, autre Suisse du $\mathrm{XvI}^{\mathrm{e}}$ siècle. Les auteurs de l' «Histoire naturelle d'un chirurgien» ne manquent pas de souligner l'absence d'archives sur des points importants de leurs analyses, confinant leur étude à une synthèse peu convaincante de la littérature secondaire, rare en ce qui concerne la Suisse romande, sans qu'on comprenne véritablement la place de la chirurgie genevoise dans une Europe en profond changement tant sur le plan politique que sur le plan intellectuel et technique. On aurait préféré un véritable commentaire composé des restes archivistiques, qui aurait permis un éclairage, malheureusement inexistant, des deux textes inédits en fin d'ouvrage. De fait, dans ce livre qui conte essentiellement le Jurine de l'histoire naturelle, la relative absence d'articulation entre les pratiques et les savoirs du chirurgien d'une part, en dehors de celles que nous avons soulignées plus haut, et l'œuvre du naturaliste d'autre part, est à déplorer. De toute façon, le lecteur peut légitimement douter du «modèle historiographique» que devrait constituer le chirurgien.

Soulignons une dernière fois les grandes qualités de Jurine, chirurgien et naturaliste. Fruit d'un travail collectif, ce livre renoue avec un principe d'historien souvent méprisé aujourd'hui : le travail rigoureux de recherche et de lecture critique d'archives. En effet, l'érudition reste un motto des études, que celle de Colán Mac Arthur couronne par l'exhumation de traces de lectures contemporaines, dans l'intention de reconstituer un manuscrit aujourd'hui disparu sur l'audition des chauves-souris. Certes, l'ouvrage n'est pas sans limites, qui sont celles de ses qualités : inégalités d'un travail véritablement collectif; effacement des pratiques du chirurgien, derrière celles - plus significatives? - du naturaliste; enfin, et problème non des moindres, tension entre l'érudition, qui conduit souvent à une méconnaissance de la littérature secondaire européenne, et la volonté de justifier le modèle historiographique de Jurine, au cœur des réseaux naturalistes européens. Le lecteur ne 
s'explique guère la raison pour laquelle Jurine est placé à l'épicentre de ce réseau naturaliste et chirurgical, faute d'une exposition de la géographie des savoirs naturalistes. Il reste que l'ensemble des études, par leur diversité d'approches, leur travail impressionnant de collecte d'archives, crée un savoir historique essentiel pour la compréhension de l'histoire des sciences naturelles à la charnière entre les XVIII ${ }^{\mathrm{e}}$ et $\mathrm{XIX}^{\mathrm{e}}$ siècles. Les planches magnifiques ajoutent à la valeur esthétique et intellectuelle d'un ouvrage qui signale, par son intelligence et sa qualité historique, la vitalité de l'école genevoise d'histoire des sciences.

Christelle RABIER

Cédric CRÉMIÈRE

Bruno Neveu, Les Facultés de théologie de l'université de France (1808-1885). Préf. de Claude Goyard. Paris, Klincksieck, 1998. $16 \times 24,847$ p., bibliogr., chronol., index, ill. (Mélanges de la bibliothèque de la Sorbonne, 27).

D'un sujet mince en apparence, Bruno Neveu tire un grand livre, remarquable par la précision de la documentation, l'impartialité des analyses, et l'étendue du contexte historique dans lequel il insère sa propre recherche. Celle-ci a pris la forme d'une thèse de doctorat en droit, soutenue le 26 juin 1993 devant l'université de droit, d'économie et de sciences sociales de Paris (Paris-II) et c'est cette thèse, légèrement retouchée en fonction des suggestions de son jury, que Bruno Neveu offre aujourd'hui au lecteur. Euvre de la maturité de son auteur, cette thèse échappe d'emblée aux tâtonnements ou aux engouements des chercheurs moins expérimentés et il serait difficile de prendre en défaut l'érudition de Neveu qui utilise avec dextérité de nombreuses séries d'archives publiques ou privées, notamment la série F 17 des Archives nationales, bien connue des historiens de l'enseignement, les archives des Affaires étrangères, les archives du Vatican (Sacrée Congrégation des affaires ecclésiastiques extraordinaires), celles de l'archevêché de Paris et le fonds Maret (revenu de Rome à l'Institut catholique de Paris). À cette masse de lettres, de notes et de listes, l'enquête de l'auteur joint tous les recueils imprimés de lois, décrets et circulaires touchant l'organisation de l'enseignement supérieur sous les divers régimes que connut la France au XIX ${ }^{\mathrm{e}}$ siècle. Au cours de ses développements, il cite d'abondants passages des textes inédits particulièrement révélateurs des mentalités qu'il analyse. De plus, six annexes, atteignant un total de presque 200 pages, apportent un complément d'information sur les argumentations échangées ou suggèrent de nouvelles recherches. En effet, l'histoire intellectuelle du catholicisme français au XIX ${ }^{\mathrm{e}}$ siècle s'est surtout intéressée à de grandes figures (Lamennais, Lacordaire, Montalembert, Veuillot), en négligeant les institutions. Ce sont celles-ci qu'explore très méthodiquement l'étude de Neveu en exhumant de sa vaste documentation une histoire bien oubliée, celle des facultés de théologie catholique créées unilatéralement par le décret impérial du 17 mars 1808, confiant à l'Université le monopole de l'enseignement et instituant en son sein des facultés de théologie catholique et protestante. Le destin de ces deux catégories d'établissement universi- 
taire fut différent, parce que les Églises luthérienne et réformée exigeaient des futurs pasteurs le passage par une faculté de théologie, alors que les diocèses catholiques possédaient des séminaires pour accueillir et former les futurs prêtres, sans obligation de grade à acquérir (malgré les tentatives faites, à diverses reprises, pour lier l'obtention d'une charge ecclésiastique à la possession d'un grade universitaire en théologie). Tout au long du XIX siècle, les facultés protestantes furent traversées par le conflit interne qui opposa durablement les « libéraux » et les «évangéliques » ou orthodoxes, à propos de l'exégèse biblique et de l'histoire des dogmes. Pour les facultés catholiques, le conflit se produisit de manière répétitive, mais sous diverses formes selon les régimes politiques, entre l'Église et l'État à propos de leurs droits respectifs sur ces facultés, leurs professeurs et leurs programmes. Les autorités romaines ne voulaient pas accorder de valeur canonique aux grades des facultés de théologie de l'Université, tenues d'enseigner la doctrine gallicane résumée dans les Quatre Articles de 1682. Au fur et à mesure que l'épiscopat français basculait vers l'ultramontanisme, sa défiance envers les dites facultés augmenta. Mais cette réserve ne connaissait plus de bornes chez les hérauts du catholicisme intransigeant, qui englobaient les facultés de théologie impériales dans leur haine de l'Université. Du côté de l'État, les ministres de l'Instruction publique, quel que fût le régime, n'entendaient pas acheter la reconnaissance canonique des facultés par le Saint Siège au prix de trop grandes concessions, concernant la nomination (ou la révocation) des enseignants et les programmes d'enseignement. L'auteur suit dans le détail, avec une extrême précision, les négociations successives qui furent poursuivies entre le Saint-Siège et le gouvernement de la France, spécialement sous le Second Empire et éclaire les raisons de leur échec. Sous la Troisième République, les facultés de théologie de l'Université se virent cernées entre deux adversaires opposés sur tout le reste, mais unis pour souhaiter leur mort: les républicains laïques qui, comme Paul Bert, veulent que la puissance publique confie l'étude des religions à des institutions scientifiques, confessionnellement neutres; les catholiques intransigeants, qui ne pensent plus qu'aux possibilités nouvelles que leur offre la loi sur la liberté de l'enseignement supérieur du 12 juillet 1875 . Pour éviter une procédure législative trop lourde, les républicains règlent finalement le problème en supprimant la dotation budgétaire des facultés de théologie dans la loi de finances du 21 mars 1885, ce qui entraînait de fait la suppression de ces facultés (les facultés protestantes étaient maintenues comme nécessaires à la formation des pasteurs). Un an auparavant, le 16 juin 1884, avait disparu Henry Maret, doyen de la faculté de théologie de la Sorbonne, qui joua avec ténacité le rôle de négociateur entre le gouvernement français et les autorités romaines. Neveu explique que cette attitude coopérative était cohérente, chez lui, avec son souci de conciliation entre le catholicisme et la société issue de la Révolution française. C'est pourquoi les laïcs modérés, comme Jules Ferry, cherchèrent le plus longtemps possible à sauver les facultés de théologie de l'Université, les jugeant aptes à former un clergé ouvert aux idées modernes. De toutes ces discussions et tractations, le récit ne perd rien et ne laisse rien ignorer au lecteur.

Cette minutie documentaire pourrait causer au lecteur quelque lassitude, mais le plaisir de lire la thèse de Neveu ne s'évanouit pas. Ce qui tient d'abord à la clarté de l'écriture, qui sait jouer des possibilités du français le plus classique, restituant ainsi, jusque dans son style, la gravité majestueuse des bureaux ministériels, au temps 
de Louis-Philippe, et la solennité paternelle des admonestations pontificales ou cardinalices. Plaisir qui tient surtout, à ce que Neveu, de son propre aveu, a cherché à comprendre les motivations et la psychologie des personnages qu'il met sous nos yeux. En le lisant, nous comprenons aussi bien la cohérence interne à l'intransigeance romaine que la rigueur avec laquelle les gouvernements de la France revendiquent leurs droits régaliens sur l'Université. Il est toutefois perceptible que l'auteur nourrit une certaine sympathie à l'égard des acteurs qui, catholiques euxmêmes ou respectueux du catholicisme, se tiennent à distance de l'ultramontanisme véhément du parti de L'Univers. Neveu connaît la cour de Rome et l'air de l'Italie aussi bien que sa langue. Mais il n'empêche : il garde une certaine faveur pour le gallicanisme modéré de ces administrateurs équitables, qui pensent agir pour le bien commun en maintenant un certain contrôle de la puissance publique sur les institutions religieuses. Les excès de Louis Veuillot l'indignent visiblement et, devant ce fanatisme, il ne ressent aucune indulgence et n'a pas de mots trop durs.

En deçà de cette sympathique intuition des personnages, l'impartialité de l'étude tient à l'ampleur du champ observé. Par son premier chapitre et par ses conclusions, par nombre de comparaisons instituées au fil des pages, l'auteur insère son objet d'étude dans l'espace européen et même nord-américain, ce qui lui permet une étude différentielle des relations entre l'Église et l'État à propos de l'institution universitaire. De plus, celle-ci est ressaisie à la racine de son histoire, c'est-à-dire au XIII siècle et il ressort de cette remontée dans le temps que, créées d'abord spontanément comme universitas des communautés d'enseignants et d'étudiants, les universités médiévales ont ensuite recouru à la puissance temporelle aussi bien qu'à la puissance spirituelle pour la validation de leurs grades. C'est le poids de l'État qui s'est accru à l'époque moderne, mais elle n'a pas inventé la nécessité de l'intervention de la puissance publique pour la reconnaissance des grades universitaires.

L'étude substantielle de Neveu ne s'est pas attachée à l'étude des contenus enseignés par les professeurs des facultés de théologie impériales et l'auteur le reconnaît dans son introduction. Cependant, ses conclusions signalent comme un fait généralement reconnu que le niveau des études ecclésiastiques en France au XIX ${ }^{\mathrm{e}}$ siècle n'était pas élevé, si l'on excepte le séminaire Saint-Sulpice de Paris. Et il analyse ici l'article souvent utilisé d'Étienne Vacherot, dans la Revue des deux mondes du 15 juillet 1868. Peut-être aurait-il pu souligner l'influence stérilisante du traditionalisme catholique sur la recherche exégétique et historique.

La bibliographie est très complète. L'on ne peut y regretter que quelques absences : l'importante thèse de René Derré, malheureusement épuisée depuis longtemps (Lamennais, ses amis et le mouvement des idées à l'époque romantique, 1824-1834, Paris, Klincksieck, 1962); à la note 8, p. 570, auraient pu être mentionnés les divers volumes du Dictionnaire du monde religieux dans la France contemporaine, fort utiles pour une recherche prosopographique; le volume du centenaire de la section «Sciences religieuses » de l'École pratique des hautes études (Cent ans de sciences religieuses en France, Paris, Cerf, 1987) aide à voir la place des religions dans la vision historique des universitaires français du XIX ${ }^{e}$ siècle. Mais, quoi qu'il en soit de telle ou telle de ces sources, il reste que les jugements sur la qualité de l'enseignement, portés par adversaires et défenseurs des facultés de théologie d'État sont contradictoires, selon les textes fournis par Neveu à l'appui des 
deux thèses en présence. Une appréciation motivée de la qualité des enseignants, appuyée sur l'inspection de quelques œuvres majeures, aurait pu renforcer l'impression favorable à ces facultés. Il demeure vrai, toutefois, qu'elles furent trop obnubilées par le «panthéisme » de la philosophie moderne, pour avoir le temps d'investir leurs professeurs dans de lourds travaux d'érudition. Ne nous attardons pas sur cette limite, clairement voulue par l'auteur, pour souligner que cette thèse de droit demeure exemplaire pour les chercheurs en histoire religieuse. Elle démontre, pour qui veut entreprendre de solides études d'histoire du christianisme, la nécessité d'acquérir une réelle compétence en matière théologique et canonique, sous peine de manquer l'objet, ou de ne pas oser sortir du champ, désormais bien balisé, de «l'histoire sociale des mentalités et des croyances ».

François LAPLANCHE 